\title{
Article
}

\section{Spurious Relationships for Nearly Non-Stationary Series}

\author{
Yushan Cheng ${ }^{1}\left(\mathbb{D}\right.$, Yongchang Hui ${ }^{1}$, Michael McAleer ${ }^{2,3,4,5,6,+}$ and Wing-Keung Wong ${ }^{7,8,9, *}$ a \\ 1 School of Mathematics and Statistics, Xi'an Jiaotong University, Xi'an 710049, China; \\ yushancheng523@126.com (Y.C.); huiyc180@xjtu.edu.cn (Y.H.) \\ 2 Department of Finance, College of Management, Asia University, Taichung City 41354, Taiwan \\ 3 Department of Bioinformatics and Medical Engineering, College of Information and Electrical Engineering, \\ Asia University, Taichung City 41354, Taiwan \\ 4 Discipline of Business Analytics, University of Sydney Business School, Darlington, NSW 2006, Australia \\ 5 Econometric Institute, Erasmus School of Economics, Erasmus University Rotterdam, \\ 3062 PA Rotterdam, The Netherlands \\ 6 Department of Economic Analysis and ICAE, Complutense University of Madrid, 28040 Madrid, Spain \\ 7 Department of Finance, Fintech and Blockchain Research Center, and Big Data Research Center, \\ Asia University, 500, Lioufeng Road, Wufeng, Taichung City 41354, Taiwan \\ 8 Department of Medical Research, China Medical University Hospital, Taichung City 41354, Taiwan \\ 9 Department of Economics and Finance, The Hang Seng University of Hong Kong, Siu Lek Yuen, \\ Hong Kong, China \\ * Correspondence: wong@asia.edu.tw \\ + Shortly after finishing the first draft of this paper, Michael McAleer passed away. Deceased 8 July 2021.
}

Citation: Cheng, Yushan, Yongchang Hui, Michael McAleer, and Wing-Keung Wong. 2021. Spurious Relationships for Nearly Non-Stationary Series. Journal of Risk and Financial Management 14: 366. https://doi.org/10.3390/jrfm14080366

Academic Editors: Eleftherios I.

Thalassinos and Aviral Kumar Tiwari

Received: 27 May 2021

Accepted: 28 July 2021

Published: 9 August 2021

Publisher's Note: MDPI stays neutral with regard to jurisdictional claims in published maps and institutional affiliations.

Copyright: (c) 2021 by the authors. Licensee MDPI, Basel, Switzerland. This article is an open access article distributed under the terms and conditions of the Creative Commons Attribution (CC BY) license (https:// creativecommons.org/licenses/by/ $4.0 /)$.

\begin{abstract}
Literature shows that the regression of independent and (nearly) nonstationary time series could result in spurious outcomes. In this paper, we conjecture that under some situations, the regression of two independent and nearly non-stationary series does not have any spurious problem at all. To check whether our conjecture holds, we set up several situations and conduct simulations to justify our conjecture. Our simulations show that under some situations, the chance that the regressions being spurious is very high for all the cases simulated in our paper. Nonetheless, under some other situations, our simulation shows that the rejection rates are much smaller than the 5\% level of significance for all the cases simulated in our paper, implying that our conjecture could hold under some situations that regression of two independent and nearly non-stationary series does not have any spurious problem at all.
\end{abstract}

Keywords: cointegration; stationarity; non-stationarity; spurious problem; nearly non-stationarity

JEL Classification: C01; C15; C22; C58; C60

\section{Introduction}

Granger and Newbold (1974) and others show that regression of independent (nearly) nonstationary time series could result in spurious outcomes, Pesaran et al. (1999) and others find that a mixed integration of orders; that is, I(0) or I(1), could be cointegrated and the residual is stationary, and Westerlund (2008) documents that many studies commit a Type 1 error by failing to reject the no-cointegration hypothesis. On the other hand, Engle and Granger (1987) establish the relationship between cointegration and error correction models that first suggested in Granger (1981) and develop estimation procedures and tests for the cointegration model. In addition, Phillips (1986) develops an asymptotic theory for regressions of integrated random processes, including the spurious regressions discovered by Granger and Newbold (1974) and the cointegrating regressions developed by Engle and Granger (1987). Entorf (1997) analyses the regression of two independent random walks with drifts and shows that the convergence to pseudo true values applies to the estimation of spurious fixed-effects models. Readers may refer to Ventosa-Santaulária (2009) for an overview of spurious regression. 
Is it possible that the regression of two independent and nearly non-stationary series does not have any spurious problem? In this paper, we explore the issue. To explore the problem, we first conjecture that under some situations, regression of two independent and nearly non-stationary series does not have any spurious problem at all. To check whether the conjecture we set holds, we first generate two independent and nearly nonstationary $\operatorname{AR}(1)$ processes, $X_{t}=\alpha_{1} X_{t-1}+\varepsilon_{t}$ and $Y_{t}=\alpha_{2} Y_{t-1}+e_{t}$ with $0.9<\left|\alpha_{1}\right|,\left|\alpha_{2}\right|<1$. We then regress $Y_{t}$ on the independent $X_{t}$ to get $Y_{t}=\alpha+\beta X_{t}+u_{t}$ and check the proportion of rejecting the null hypothesis that the beta $(\beta)$ is zero. We first find that under some situations, consistent with the literature, regressing two independent and (nearly) nonstationary time series could be spurious. Nonetheless, we also find that under some other situations, different from the literature, our results show that the rejection rates are much smaller than the $5 \%$ level of significance for all the cases simulated in our paper, implying that under some other situations, regressing nearly nonstationary $Y_{t}$ on independent and nearly nonstationary $X_{t}$ will not get any spurious problem at all as shown in all the cases being simulated in our paper.

The rest of the paper is organized as follows. In Section 2, we state the basic models for the regression and the regression with a spurious problem. In Section 3, we state our model setup and construct the algorithm for the simulation. In Section 4 , we discuss our findings from our simulation and the last section concludes.

\section{The Model}

In this section, we state the basic models for a simple regression and the regression with a spurious problem. We first state the basic simple regression model.

\subsection{Linear Regression Model}

In this paper, we consider the following simple regression model:

$$
Y_{t}=\alpha+\beta X_{t}+u_{t}
$$

where $u_{t}$ is a random component denoted as error term assumed to be independent and identically distributed (iid) with mean 0 and variance $\sigma_{u}^{2}, t=1, \cdots, T$ in which $T$ is the sample size, $\alpha$ is the intercept parameter, and $\beta$ is the slope parameter.

The most important hypothesis for the simple linear regression stated in (1) is

$$
H_{0}: \beta=0 \text { versus } H_{1}: \beta \neq 0 \text {, }
$$

which is used to detect the linear relationship between two variables $Y$ and $X$. If the null hypothesis $H_{0}$ in (2) is true, then for the model in (1), the population mean of $Y_{t}$ is always equal to $\alpha$ for any value of $X_{t}$, concluding that $Y_{t}$ does not depend on the value of $X_{t}$ and there is no linear relationship between $X_{t}$ and $Y_{t}$. On the other hand, if the alternative hypothesis $H_{1}$ is true, then one could conclude that a change in $X_{t}$ is associated with a change in $Y_{t}$ linearly.

To test whether the null hypothesis $H_{0}$ in (2) is true, one could use the following $T$ test:

$$
T=\frac{\hat{\beta}-\beta}{S E(\hat{\beta})}=\frac{\hat{\beta}}{S E(\hat{\beta})},
$$

where

$$
\hat{\beta}=\frac{\sum_{t=1}^{N}\left(X_{t}-\bar{X}\right)\left(Y_{t}-\bar{Y}\right)}{\sum_{t=1}^{N}\left(X_{t}-\bar{X}\right)^{2}},
$$

is the estimate of $\beta$ in which $\bar{X}=\sum_{t=1}^{T} X_{t} / T, \bar{Y}=\sum_{t=1}^{T} Y_{t} / T$, and

$$
S E(\hat{\beta})=\sqrt{\frac{\sum_{t=1}^{T}\left(\hat{u}_{t}^{2}\right) /(T-2)}{\sum_{t=1}^{T}\left(X_{t}-\bar{X}\right)^{2}}}
$$


is the standard error of the estimate measuring the accuracy of prediction with $\hat{u}_{t}=\left(Y_{t}-\hat{\alpha}-\hat{\beta} X_{t}\right)^{2}$. It is well known that the test statistic $T$ follows a $t$-distribution with $T-2$ degrees of freedom if the null hypothesis $H_{0}$ is true. We note that Goodness of fit can refer to specific individual factors (or variables) in the regression equation, whereas R-squared refers to a specific numerical value of the entire regression model.

\subsection{Spurious Regression}

A spurious relationship is a relationship that does not make sense. In this situation, two or more independent variables could appear to be correlated with the effect of an unseen factor ("confounding factor" or "lurking variable"). That is, goodness-of-fit indicators from regression such as $R^{2}$ are likely to be large, implying a valid fit even if the underlying variables are not truly related.

A variable, say, $Y_{t}$, is said to be integrated of order $d$, and denoted as $Y_{t}=I(d)$, if it has a stationary, invertible, and stochastic ARMA representation after differencing $d$ times, it is stationary if $d<1$, is non-stationary if $d \geq 1$, and is nearly non-stationary if $d<1$ but close to 1. Granger and Newbold (1974) and others find that if

$$
Y_{t} \sim I(1) \text { and } X_{t} \sim I(1)
$$

then regression in (1) could be spurious. It means that even when $Y_{t}$ and $X_{t}$ are known to be independent, applying the test statistic $T$ in (3) to an ordinary least square regression could misleadingly indicate a good fit when non-stationary time series data are involved. Granger and Newbold (1974) exhibit an example in which an equation has $R^{2}=0.997$ and the value of the Durbin-Watson (DW) statistic is 0.093, implying that when the residuals are strongly autocorrelated in time series regression, the interpretability of the coefficients could be questionable.

Since Granger and Newbold (1974) and others show that regression of independent and nonstationary time series could result in spurious outcomes, it is common to believe that regression of independent and nearly nonstationary time series could also get spurious outcomes. In this paper, we believe that there are some cases in which regression of independent and nearly nonstationary time series may not be spurious. Thus, we set up the following conjecture:

Conjecture 1. Under some situations, the regression of two independent and nearly non-stationary series does not have any spurious problem at all.

To examine whether the above conjecture could hold in some situations, we will discuss it in the next section.

\section{Model Setup and Algorithm}

In this section, we first state the model setting of generating two purely independent and nearly nonstationary time series, regressing one of them onto the other, and examining whether the corresponding regression is spurious. We then construct the algorithm for the simulation and discuss our simulation result in the next section.

\subsection{Model Setup}

We consider the simple linear regression in (1) between two unrelated nearly nonstationary $\operatorname{AR}(1)$ series $X_{t}$ and $Y_{t}$ such that

$$
X_{t}=\alpha_{1} X_{t-1}+\varepsilon_{t} \text { and } Y_{t}=\alpha_{2} Y_{t-1}+e_{t} \text {, with } \varepsilon_{t} \stackrel{i i d}{\sim}\left(0, \sigma_{\varepsilon}^{2}\right) \text { and } e_{t} \stackrel{i i d}{\sim}\left(0, \sigma_{e}^{2}\right)
$$

in which $0.9<\left|\alpha_{i}\right|<1(i=1,2)$. For simplicity, we assume that both $\varepsilon_{t}$ and $e_{t}$ follow:

$$
f(a ; p) \propto \frac{1}{\sigma}\left\{1+\frac{a^{2}}{k \sigma^{2}}\right\}^{-p} \quad(-\infty<a<\infty)
$$


where $k=2 p-1$ and $p \geq 2$. We note that $E(a)=0, V(a)=\sigma^{2}$, and $t=\sqrt{(k / v)}(a / \sigma)$ follows a Student's $t$ distribution with $v=2 p-1$ degrees of freedom (df). For $1 \leq p<2$, $k$ is equated to 1 and in this case, $\sigma$ in (8) is simply a scale parameter. When $v=1$, it becomes a Cauchy distribution and when $v=\infty$, it becomes a normal distribution. Readers may refer to Pötzelberger (1990), Tiku and Wong (1998), Tiku et al. (1999, 2000), Wong and Bian (2005), Fu and Fu (2015), and others to know more properties of AR(1) series.

To simulate $X_{t}$ and $Y_{t}$ properly, without loss of generality, we will consider different factors that could affect the behavior of the time series. First, we consider the distribution of the error terms. We choose a time series that follows the following four different iid error distributions in our study:

Situation 1. We assume that the distribution of the error terms $\varepsilon_{t}$ and $e_{t}$ defined in (7) follow the following situations:

1. a standard normal distribution: that is, both $\varepsilon_{t}$ and $e_{t} \sim N(0,1)$;

2. a t-distribution with $d f=5$ : that is, both $\varepsilon_{t}$ and $e_{t} \sim t(5)$;

3. at-distribution with $d f=2$ : that is, both $\varepsilon_{t}$ and $e_{t} \sim t(2)$; and

4. at-distribution with $d f=1$ : that is, both $\varepsilon_{t}$ and $e_{t}$ follow the standard Cauchy distribution.

Second, we vary the lengths of the times series and simulate a time series with the following four different lengths in our study as stated in the following situations:

Situation 2. We consider that the lengths of the times series $Y_{t}$ and $X_{t}$ defined in (7) to be: (i) $T=100$; (ii) $T=200$; (iii) $T=400$; and (iv) $T=800$.

After deciding the error distribution and the lengths of the AR(1) processes, we now consider the different values of $\alpha_{1}$ and $\alpha_{2}$. In our model, since both $X_{t}$ and $Y_{t}$ are nearly nonstationary, we choose $0.9<\left|\alpha_{i}\right|<1(i=1,2)$ and, in particular, we define $A^{-}=\{-0.99,-0.97,-0.95,-0.92,-0.9\}$ and $A^{+}=\{0.99,0.97,0.95,0.92,0.9\}^{1}$ and consider the following values for both $\alpha_{1}$ and $\alpha_{2}$ as stated in Situations 3 and 4:

Situation 3. We consider that the values of both $\alpha_{1}$ and $\alpha_{2}$ such that $\alpha_{1} \in A^{+}$and $\alpha_{2} \in A^{-}$.

Situation 4. We consider that the values of both $\alpha_{1}$ and $\alpha_{2}$ such that $\alpha_{1} \in A^{-}$and $\alpha_{2} \in A^{+}$.

We note that in this paper, we consider Situations 3 and 4 because when two autoregressive processes in which one is associated to the zero frequency; that is, the AR(1) with a positive coefficient in our paper, and the other is associated to the Nyquist frequency $(\pi)$; that is, the AR(1) with a negative coefficient in our paper that has power at frequency $\pi$ and completes a cycle every 2 observations, are independent or even asymptotically orthogonal. Readers may refer to Johansen and Schaumburg (1999), Ghysels and Osborn (2001), and del Barrio Castro et al. $(2018,2019)$ for more information. Readers may also refer to seasonal unit root tests, see, for example, del Barrio Castro et al. (2012) and Smith et al. (2009), and cointegration for processes integrated at different frequencies, see, for example, del Barrio Castro et al. (2020) with properties that are related to the series we are using in our paper. ${ }^{2}$

With four different error distributions, four different time series lengths, and the above 50 combinations of $\alpha_{1}$ and $\alpha_{2}$ values as stated in Assumptions 1, 2, 3, and 4, there are in total 800 cases of simulation in our study for the cases when autoregressive coefficients $\alpha_{1}$ and $\alpha_{2}$ have different signs.

Nevertheless, in this paper, we also study the cases when both autoregressive coefficients $\alpha_{1}$ and $\alpha_{2}$ are of the same signs, either positive or negative. Thus, we include the following situations in our study:

Situation 5. We consider that the values of both $\alpha_{1}$ and $\alpha_{2}$ such that $\alpha_{1} \in A^{+}$and $\alpha_{2} \in A^{+}$. 
Situation 6. We consider that the values of both $\alpha_{1}$ and $\alpha_{2}$ such that $\alpha_{1} \in A^{-}$and $\alpha_{2} \in A^{-}$.

\subsection{Algorithm}

The two series $X_{t}$ and $Y_{t}$ are generated from independent error terms, and thus, they are expected not to be related. However, Granger and Newbold (1974) and others have shown that regression of independent nonstationary time series could result in spurious outcomes. In this paper, we believe that it is possible that when regressinng independent and nearly nonstationary $Y_{t}$ and $X_{t}$ as shown in Equation (1) may not be spurious under some situations as we stated in Conjecture 1. To check whether Conjecture 1 could hold under some situations, we set the following algorithm for each situation (different error distributions, different time series lengths, different combinations of $\alpha_{1}$ and $\alpha_{2}$ ) as described in Section 3.1:

Algorithm 1: For each situation (different error distributions, different time series lengths, different combinations of $\alpha_{1}$ and $\alpha_{2}$ ) as described in Section 3.1, we will conduct the following steps in our simulation:

1. Simulate 10,000 pairs of $X_{t}$ and $Y_{t}$ defined in (7) with coefficients described in Section 3.1.

2. For each pair of simulated $X_{t}$ and $Y_{t}$, fit model in (1). Thus, in each subcase, we will obtain 10,000 $\hat{\beta}^{\prime}$ s and 10,000 corresponding $p$-values.

3. Plot the distribution of $\hat{\beta}^{\prime}$ s and record the standard errors and t-statistics of $\hat{\beta}$.

4. Use the $T$ test defined in Equation (3) to test whether the null hypothesis $H_{0}$ in (2) hold. $H_{0}$ is rejected if $p$-value for the $T$ test is less than 0.05 . Calculate the proportion of significant $\hat{\beta}^{\prime}$ s or proportion of $p$-values that are less than 0.05 among the 10,000 fitted linear regression models in each subcase. This proportion is denoted as the rejection rate in this paper.

For each situation (different error distributions, different time series lengths, different combinations of $\alpha_{1}$ and $\alpha_{2}$ ) as described in Section 3.1, we will conduct simulation as described in Algorithm 1 and discuss the results in the next section.

\section{Simulation}

We follow Algorithm 1 to conduct simulation for each situation (different error distributions, different time series lengths, different combinations of $\alpha_{1}$ and $\alpha_{2}$ ) as described in Section 3.1. The simulation helps us to examine whether the $T$ statistic as shown in Equation (3) for the model as shown in Equation (1) follow a Student $t$-distribution. If $X_{t}$ and $Y_{t}$ are unrelated, the true null hypothesis that all $\beta$ coefficients are zero should be rejected around $5 \%$ of the time at the significance level of $5 \%$. If the $\mathrm{T}$ test is good, that is, $\hat{\beta}^{\prime}$ s follow student $t$-distribution, the rejection rate should be close to $5 \%$. If the rejection rate is significantly greater than $5 \%$, then we conclude that there exists the spurious problem. In addition, we believe that it is possible that when regressing independent and nearly nonstationary $Y_{t}$ and $X_{t}$ as shown in (1) may not be spurious under some situations as we hypothesized in Conjecture 1 . To check whether Conjecture 1 could hold under some situations, we discuss it in this section. We first discuss the results of the simulation for the cases when $\alpha_{1}$ and $\alpha_{2}$ are of different signs in the next subsection.

\subsection{Simulation for the Cases When $\alpha_{1}$ and $\alpha_{2}$ Are of Different Signs}

We first analyze cases as stated in Situation 3 and exhibit the results in Tables A1-A4 displaying in Appendix A that report the rejecting frequency of the $T$ test when $\left(\alpha_{1}, \alpha_{2}\right) \in$ $\left(A^{+}, A^{-}\right)$. From Tables A1-A4, one can observe that when choosing the values of both $\alpha_{1}$ and $\alpha_{2}$ as stated in Situation 3 are from $|0.9|$ to $|0.99|$, the rejection rate is about 0.0000 for any $n$ and for any error distribution studied in our paper, except the situation when the error term follows a $t(1)$ in which the rejection rates are close to 0.0004 . 
We then analyze the cases as stated in Situation 4 and show the results in Tables A5-A8 displaying in Appendix $B$ that report the rejecting frequency of the $T$ test when $\left(\alpha_{1}, \alpha_{2}\right) \in$ $\left(A^{-}, A^{+}\right)$. Similarly, from Tables A5-A8, one can observe that when choosing values of both $\alpha_{1}$ and $\alpha_{2}$ as stated in Situation 4 are between $|0.9|$ and $|0.99|$, the rejection rate is zero or close to zero for any $n$ and any error distribution studied in our paper.

Our analysis shows that for all the cases when choosing values for $\left(\alpha_{1}, \alpha_{2}\right)$ as stated in Situations 3 and 4 and when choosing values of both $\left|\alpha_{1}\right|$ and $\left|\alpha_{2}\right|$ are between 0.9 and 0.99 , the rejection rates are much smaller than the $5 \%$ level of significance, implying that when $\left(\alpha_{1}, \alpha_{2}\right)$ follow Situations 3 and 4 and when both $\left|\alpha_{1}\right|$ and $\left|\alpha_{2}\right|$ are between 0.9 and 0.99 , all the corresponding regressions do not encounter any spurious problem for all the cases simulated in our paper, confirming that Conjecture 1 holds. In other words, our analysis shows that when independent $Y_{t}$ and $X_{t}$ follow nearly nonstationary AR(1) model and the autoregressive coefficients $\alpha_{1}$ and $\alpha_{2}$ have opposite signs, there is no spurious problem in the regression stated in Equation (1) and Conjecture 1 holds.

\subsection{Simulation for the Cases When $\alpha_{1}$ and $\alpha_{2}$ Are of the Same Sign}

We turn to examine whether the regression shown in Equation (1) is spurious for the cases when both $\alpha_{1}$ and $\alpha_{2}$ are of the same signs; that is, both $\alpha_{1}$ and $\alpha_{2}$ are positive or both are negative. To do so, we follow Algorithm 1 to conduct simulations for the cases when both $\alpha_{1}$ and $\alpha_{2}$ are positive and both are negative as displayed in Situations 5 and 6 and exhibit the results in Tables A9-A16 displaying in Appendices C and D, respectively.

We first discuss the cases when both $\alpha_{1}$ and $\alpha_{2}$ are positive as stated in Situation 5 . Compared with the results in Tables A1-A8, all of the rejection rates in Tables A9-A12 are significantly higher than $5 \%$ and the rejecting frequency of the $T$ test is higher than $49 \%$ for any $n$ and any error distribution studied in our paper, except the situation when the error term follows $t(1)$ in which the rejection rates is higher than $32 \%$. In addition, as $n$ increases, or either $\alpha_{1}$ or $\alpha_{2}$ increases, or as the error distributions are further away from normal distribution, the rejecting rate increases even further.

We turn to discuss the cases when both $\alpha_{1}$ and $\alpha_{2}$ are negative as stated in Situation 6 . Similar to the cases when both $\alpha_{1}$ and $\alpha_{2}$ are positive, when both $\alpha_{1}$ and $\alpha_{2}$ are negative, the rejecting frequency of the $T$ test is higher than $50 \%$ for any $n$ and for any error distribution studied in our paper, except the situation when the error term follows $t(1)$ in which the rejection rates is higher than $31 \%$. In addition, Similar to the cases when both $\alpha_{1}$ and $\alpha_{2}$ are positive, as $n$ increases, or either $\alpha_{1}$ or $\alpha_{2}$ increases, or as the error distributions are further away from normal distribution, the rejecting rate increases even further.

Our analysis shows that, different from all the cases when for $\alpha_{1}$ and $\alpha_{2}$ are of different signs, for all the cases when $\alpha_{1}$ and $\alpha_{2}$ are of the same signs, either positive or negative, as stated in Situations 5 and 6, respectively, and when both $\left|\alpha_{1}\right|$ and $\left|\alpha_{2}\right|$ are between 0.9 and 0.99 , the rejection rate is much higher than the $5 \%$ level of significance for all the cases studied in our paper and it could be higher than $49 \%$, implying that when $\left(\alpha_{1}, \alpha_{2}\right)$ follow Situations 5 and 6 and when both $\left|\alpha_{1}\right|$ and $\left|\alpha_{2}\right|$ are between 0.9 and 0.99 , the chance that the regressions being spurious is very high for all the cases simulated in our paper, which, in turn, rejects Conjecture 1 for all the cases in Situations 5 and 6.

\section{Concluding Remarks}

In this paper, we conjecture that under some situations, the regression of two independent and nearly non-stationary series does not have any spurious problem at all. To check whether our conjecture holds, we first generate two independent and nearly nonstationary AR(1) processes, $X_{t}=\alpha_{1} X_{t-1}+\varepsilon_{t}$ and $Y_{t}=\alpha_{2} Y_{t-1}+e_{t}$ in which $0.9<\left|\alpha_{1}\right|,\left|\alpha_{2}\right|<1$. We then regress $Y_{t}$ on independent $X_{t}$ to get $Y_{t}=\alpha+\beta X_{t}+u_{t}$ and check whether the proportion of rejecting the null hypothesis of the beta $(\beta)$ to be zero. We first find that consistent with the literature that supports the hypothesis of regressing two independent and (nearly) nonstationary time series could be spurious, when both $\alpha_{1}$ and $\alpha_{2}$ are of the same signs, either positive or negative, and when the values of both $\left|\alpha_{1}\right|$ and $\left|\alpha_{2}\right|$ are 
between 0.9 and 0.99 , the rejection rate is much bigger than the $5 \%$ level of significance in all the cases examined in our simulation and it could be higher than $49 \%$ in many cases, implying that the chance that the regressions being spurious is very high for all the cases when both $\alpha_{1}$ and $\alpha_{2}$ are of the same signs.

Nonetheless, for all the cases when for $\alpha_{1}$ and $\alpha_{2}$ are of different signs, then different from the literature, our results show that when both $\left|\alpha_{1}\right|$ and $\left|\alpha_{2}\right|$ are between 0.9 and 0.99 , the rejection rates are much smaller than the $5 \%$ level of significance for all the cases studied in our paper, implying that when $\alpha_{1}$ and $\alpha_{2}$ are of different signs, regressing nearly nonstationary $Y_{t}$ on independent and nearly nonstationary $X_{t}$ will not get any spurious problem at all for all the cases being simulated in our paper.

We note that the literature shows that the regression of independent and (nearly) nonstationary time series could result in spurious outcomes. In this paper, we conjecture that under some situations, regression of two independent and nearly non-stationary series does not have any spurious problem at all, and in this paper, we aim to find some situations that our conjecture could hold. In this paper, we find that when $\left(\alpha_{1}, \alpha_{2}\right) \in\left(A^{+}, A^{-}\right)$or $\left(A^{-}, A^{+}\right)$, then our conjecture holds. We note that when $\left(\alpha_{1}, \alpha_{2}\right) \in\left(A^{+}, A^{-}\right)$or $\left(A^{-}, A^{+}\right)$, our conjecture holds which does not imply that these are only situations that our conjecture holds. There could have other situations that our conjecture could hold. We leave it to future studies to find other situations that our conjecture could hold. The purpose of our paper is to tell readers that when one finds regression of any two or more time series that do not have any spurious problem, this does not necessarily imply that the series are not independent. Thus, academics and practitioners should conduct some proper tests to show whether the series are independent.

Some academics may wonder whether there are some financial or economic time series that exhibit extreme negative autocorrelations. We believe there could have some financial or economic time series exhibit positive autocorrelations and some exhibit negative autocorrelations. We note that the time period used in our paper may not be daily or monthly, it should be set to fit the nature of the time series. It is well-known that stock returns could be overreacted or underreacted, this means that it could be positively auto-correlated or negatively auto-correlated and the true unobserved stock returns are positively auto-correlated or negatively auto-correlated. Whether they are extreme positively auto-correlated or negatively auto-correlated will depend on particular stocks. In addition, as we have mentioned before, when $\left(\alpha_{1}, \alpha_{2}\right) \in\left(A^{+}, A^{-}\right)$or $\left(A^{-}, A^{+}\right)$, our conjecture holds which does not imply that these are the only situations that our conjecture could hold. There may have other situations that our conjecture could hold. Some financial or economic time series could follow other situations that yet to be discovered, and thus, the conjecture could be important not only for statistics, but also for economics and finance. We also note that in our paper, we only consider $\left(A^{+}, A^{-}\right)$to cover nearly non-stationary series but do not cover the situations $A^{+}=1$ and $A^{-}=-1$. We do not cover the situation $A^{+}=1$ because this has been well-studied in the literature. On the other hand, we do not cover the situation $A^{-}=-1$ because this situation, we believe, is of no practice relevance.

We note that as far as we know, this paper is the first paper to discover that under some situations, the regression of two independent and nearly non-stationary series does not have any spurious problem at all. We follow Granger and Newbold (1974) and others to provide simulation results to show our discovery. Academics could follow Phillips (1986), Johansen and Schaumburg (1999), and others to provide formal proof of the finding in our paper to replace Brownian motions by using the OU processes with $\exp (c / T)$ to approximate $(1+c / T) .{ }^{3}$ We will leave it to further research to develop the theoretical results to explain the phenomena discovered in this paper. We also note that in this paper, we get very good results by using $0.9<|\alpha|<1$. One may get good results by using the near-integrated approach. We will leave this to future studies. ${ }^{4}$ Another problem in our study is that there is a serious problem with under-rejection. Further study could expose this problem and correct the test properly. 
Author Contributions: Conceptualization and methodology, Y.C., Y.H. and W.-K.W.; formal analysis, Y.C., Y.H. and W.-K.W.; writing-original draft preparation, Y.C., Y.H., M.M., and W.-K.W.; writing-review and editing, Y.C., Y.H., M.M. and W.-K.W. project administration and funding, Y.C., Y.H., M.M. and W.-K.W. All authors have read and agreed to the published version of the manuscript.

Funding: This research has been supported by the Asia University, China Medical University Hospital, The Hang Seng University of Hong Kong, Research Grants Council (RGC) of Hong Kong (project number 12500915), and Ministry of Science and Technology (MOST, Project Numbers 1062410-H-468-002 and 107-2410-H-468-002-MY3), Taiwan.

Acknowledgments: The authors are grateful to the anonymous referees for substantive comments that have significantly improved this manuscript. For financial support, the third author wishes to thank the Australian Research Council and the National Science Council, Ministry of Science and Technology (MOST), Taiwan. The fourth author acknowledges the Research Grants Council of Hong Kong (Project Numbers 12500915), Ministry of Science and Technology, Taiwan (MOST, Project Numbers 106-2410-H468-002 and 107-2410-H-468-002-MY3), Asia University, China Medical University Hospital, and The Hang Seng University of Hong Kong. The fourth author would also like to thank Robert B. Miller and Howard E. Thompson for their continuous guidance and encouragement.

Conflicts of Interest: The authors declare no conflict of interest.

Appendix A. $\left(\alpha_{1}, \alpha_{2}\right) \in\left(A^{+}, A^{-}\right)$

Table A1. Rejection rate, error $\sim \mathrm{N}(0,1)$.

\begin{tabular}{|c|c|c|c|c|c|c|c|}
\hline$\alpha_{2}$ & $\alpha_{1}$ & $n=100$ & $n=200$ & $n=400$ & $n=800$ & Raverage & Saverage \\
\hline \multirow{5}{*}{-0.9} & 0.9 & 0.0000 & 0.0000 & 0.0000 & 0.0000 & 0.0000 & \multirow{5}{*}{0.0000} \\
\hline & 0.92 & 0.0000 & 0.0000 & 0.0000 & 0.0000 & 0.0000 & \\
\hline & 0.95 & 0.0000 & 0.0000 & 0.0000 & 0.0000 & 0.0000 & \\
\hline & 0.97 & 0.0000 & 0.0000 & 0.0000 & 0.0000 & 0.0000 & \\
\hline & 0.99 & 0.0000 & 0.0000 & 0.0000 & 0.0000 & 0.0000 & \\
\hline \multicolumn{2}{|c|}{ Caverage } & 0.0000 & 0.0000 & 0.0000 & 0.0000 & 0.0000 & 0.0000 \\
\hline \multirow{5}{*}{-0.92} & 0.9 & 0.0000 & 0.0000 & 0.0000 & 0.0000 & 0.0000 & \multirow{5}{*}{0.0000} \\
\hline & 0.92 & 0.0000 & 0.0000 & 0.0000 & 0.0000 & 0.0000 & \\
\hline & 0.95 & 0.0000 & 0.0000 & 0.0000 & 0.0000 & 0.0000 & \\
\hline & 0.97 & 0.0000 & 0.0000 & 0.0000 & 0.0000 & 0.0000 & \\
\hline & 0.99 & 0.0000 & 0.0000 & 0.0000 & 0.0000 & 0.0000 & \\
\hline \multicolumn{2}{|c|}{ Caverage } & 0.0000 & 0.0000 & 0.0000 & 0.0000 & 0.0000 & 0.0000 \\
\hline \multirow{5}{*}{-0.95} & 0.9 & 0.0000 & 0.0000 & 0.0000 & 0.0000 & 0.0000 & \multirow{5}{*}{0.0000} \\
\hline & 0.92 & 0.0000 & 0.0000 & 0.0000 & 0.0000 & 0.0000 & \\
\hline & 0.95 & 0.0000 & 0.0000 & 0.0000 & 0.0000 & 0.0000 & \\
\hline & 0.97 & 0.0000 & 0.0000 & 0.0000 & 0.0000 & 0.0000 & \\
\hline & 0.99 & 0.0000 & 0.0000 & 0.0000 & 0.0000 & 0.0000 & \\
\hline \multicolumn{2}{|c|}{ Caverage } & 0.0000 & 0.0000 & 0.0000 & 0.0000 & 0.0000 & 0.0000 \\
\hline \multirow{5}{*}{-0.97} & 0.9 & 0.0000 & 0.0000 & 0.0000 & 0.0000 & 0.0000 & \multirow{5}{*}{0.0000} \\
\hline & 0.92 & 0.0000 & 0.0000 & 0.0000 & 0.0000 & 0.0000 & \\
\hline & 0.95 & 0.0000 & 0.0000 & 0.0000 & 0.0000 & 0.0000 & \\
\hline & 0.97 & 0.0000 & 0.0000 & 0.0000 & 0.0000 & 0.0000 & \\
\hline & 0.99 & 0.0000 & 0.0000 & 0.0000 & 0.0000 & 0.0000 & \\
\hline \multicolumn{2}{|c|}{ Caverage } & 0.0000 & 0.0000 & 0.0000 & 0.0000 & 0.0000 & 0.0000 \\
\hline
\end{tabular}


Table A1. Cont.

\begin{tabular}{|c|c|c|c|c|c|c|c|}
\hline$\alpha_{2}$ & $\alpha_{1}$ & $n=100$ & $n=200$ & $n=400$ & $n=800$ & Raverage & Saverage \\
\hline \multirow{5}{*}{-0.99} & 0.9 & 0.0000 & 0.0000 & 0.0000 & 0.0000 & 0.0000 & \multirow{5}{*}{0.0000} \\
\hline & 0.92 & 0.0000 & 0.0000 & 0.0000 & 0.0000 & 0.0000 & \\
\hline & 0.95 & 0.0000 & 0.0000 & 0.0000 & 0.0000 & 0.0000 & \\
\hline & 0.97 & 0.0000 & 0.0000 & 0.0000 & 0.0000 & 0.0000 & \\
\hline & 0.99 & 0.0000 & 0.0000 & 0.0000 & 0.0000 & 0.0000 & \\
\hline \multicolumn{2}{|c|}{ Caverage } & 0.0000 & 0.0000 & 0.0000 & 0.0000 & 0.0000 & 0.0000 \\
\hline
\end{tabular}

Note: 'Raverage' (stands for row average) is the average rejection rate for different values of $n$ for the same case; 'Caverage' (stands for column average) is the average rejection rate for different cases in which $\alpha_{1} \in A^{+}=$ $\{0.99,0.97,0.95,0.92,0.9\}$ for the same $n$ for the same Situation; 'Saverage' (stands for Situation average) is the average rejection rate for different cases in which $\alpha_{1} \in A^{+}=\{0.99,0.97,0.95,0.92,0.9\}$ and different $n$ for the same situation and 'overall average' in the 3 to 6 columns is the overall average for each $n$ for all situations and all cases while 'overall average' in the last two column is the overall average for all the cases in the entire table.

Table A2. Rejection rate, error $\sim \mathrm{t}(5)$.

\begin{tabular}{|c|c|c|c|c|c|c|c|}
\hline$\alpha_{2}$ & $\alpha_{1}$ & $n=100$ & $n=200$ & $n=400$ & $n=800$ & Raverage & Saverage \\
\hline \multirow{5}{*}{-0.9} & 0.9 & 0.0000 & 0.0000 & 0.0000 & 0.0000 & 0.0000 & \multirow{5}{*}{0.0000} \\
\hline & 0.92 & 0.0000 & 0.0000 & 0.0000 & 0.0000 & 0.0000 & \\
\hline & 0.95 & 0.0000 & 0.0000 & 0.0000 & 0.0000 & 0.0000 & \\
\hline & 0.97 & 0.0000 & 0.0000 & 0.0000 & 0.0000 & 0.0000 & \\
\hline & 0.99 & 0.0000 & 0.0000 & 0.0000 & 0.0000 & 0.0000 & \\
\hline \multicolumn{2}{|c|}{ Caverage } & 0.0000 & 0.0000 & 0.0000 & 0.0000 & 0.0000 & 0.0000 \\
\hline \multirow{5}{*}{-0.92} & 0.9 & 0.0000 & 0.0000 & 0.0000 & 0.0000 & 0.0000 & \multirow{5}{*}{0.0000} \\
\hline & 0.92 & 0.0000 & 0.0000 & 0.0000 & 0.0000 & 0.0000 & \\
\hline & 0.95 & 0.0000 & 0.0000 & 0.0000 & 0.0000 & 0.0000 & \\
\hline & 0.97 & 0.0000 & 0.0000 & 0.0000 & 0.0000 & 0.0000 & \\
\hline & 0.99 & 0.0000 & 0.0000 & 0.0000 & 0.0000 & 0.0000 & \\
\hline \multicolumn{2}{|c|}{ Caverage } & 0.0000 & 0.0000 & 0.0000 & 0.0000 & 0.0000 & 0.0000 \\
\hline \multirow{5}{*}{-0.95} & 0.9 & 0.0000 & 0.0000 & 0.0000 & 0.0000 & 0.0000 & \multirow{5}{*}{0.0000} \\
\hline & 0.92 & 0.0000 & 0.0000 & 0.0000 & 0.0000 & 0.0000 & \\
\hline & 0.95 & 0.0000 & 0.0000 & 0.0000 & 0.0000 & 0.0000 & \\
\hline & 0.97 & 0.0000 & 0.0000 & 0.0000 & 0.0000 & 0.0000 & \\
\hline & 0.99 & 0.0000 & 0.0000 & 0.0000 & 0.0000 & 0.0000 & \\
\hline \multicolumn{2}{|c|}{ Caverage } & 0.0000 & 0.0000 & 0.0000 & 0.0000 & 0.0000 & 0.0000 \\
\hline \multirow{5}{*}{-0.97} & 0.9 & 0.0000 & 0.0000 & 0.0000 & 0.0000 & 0.0000 & \multirow{5}{*}{0.0000} \\
\hline & 0.92 & 0.0000 & 0.0000 & 0.0000 & 0.0000 & 0.0000 & \\
\hline & 0.95 & 0.0000 & 0.0000 & 0.0000 & 0.0000 & 0.0000 & \\
\hline & 0.97 & 0.0000 & 0.0000 & 0.0000 & 0.0000 & 0.0000 & \\
\hline & 0.99 & 0.0000 & 0.0000 & 0.0000 & 0.0000 & 0.0000 & \\
\hline \multicolumn{2}{|c|}{ Caverage } & 0.0000 & 0.0000 & 0.0000 & 0.0000 & 0.0000 & 0.0000 \\
\hline \multirow{5}{*}{-0.99} & 0.9 & 0.0000 & 0.0000 & 0.0000 & 0.0000 & 0.0000 & \multirow{5}{*}{0.0000} \\
\hline & 0.92 & 0.0000 & 0.0000 & 0.0000 & 0.0000 & 0.0000 & \\
\hline & 0.95 & 0.0000 & 0.0000 & 0.0000 & 0.0000 & 0.0000 & \\
\hline & 0.97 & 0.0000 & 0.0000 & 0.0000 & 0.0000 & 0.0000 & \\
\hline & 0.99 & 0.0000 & 0.0000 & 0.0000 & 0.0000 & 0.0000 & \\
\hline \multicolumn{2}{|c|}{ Caverage } & 0.0000 & 0.0000 & 0.0000 & 0.0000 & 0.0000 & 0.0000 \\
\hline
\end{tabular}

Note: 'Raverage' (stands for row average) is the average rejection rate for different values of $n$ for the same case; 'Caverage' (stands for column average) is the average rejection rate for different cases in which $\alpha_{1} \in A^{+}=$ $\{0.99,0.97,0.95,0.92,0.9\}$ for the same $n$ for the same Situation; 'Saverage' (stands for Situation average) is the average rejection rate for different cases in which $\alpha_{1} \in A^{+}=\{0.99,0.97,0.95,0.92,0.9\}$ and different $n$ for the same situation and 'overall average' in the 3 to 6 columns is the overall average for each $n$ for all situations and all cases while 'overall average' in the last two column is the overall average for all the cases in the entire table. 
Table A3. Rejection rate, error $\sim \mathrm{t}(2)$.

\begin{tabular}{|c|c|c|c|c|c|c|c|}
\hline$\alpha_{2}$ & $\alpha_{1}$ & $n=100$ & $n=200$ & $n=400$ & $n=800$ & Raverage & Saverage \\
\hline \multirow{5}{*}{-0.9} & 0.9 & 0.0000 & 0.0001 & 0.0000 & 0.0001 & 0.0001 & \multirow{5}{*}{0.0000} \\
\hline & 0.92 & 0.0001 & 0.0000 & 0.0000 & 0.0000 & 0.0001 & \\
\hline & 0.95 & 0.0000 & 0.0001 & 0.0000 & 0.0000 & 0.0000 & \\
\hline & 0.97 & 0.0001 & 0.0000 & 0.0000 & 0.0000 & 0.0000 & \\
\hline & 0.99 & 0.0000 & 0.0000 & 0.0000 & 0.0000 & 0.0000 & \\
\hline \multicolumn{2}{|c|}{ Caverage } & 0.0000 & 0.0000 & 0.0000 & 0.0000 & 0.0000 & 0.0000 \\
\hline \multirow{5}{*}{-0.92} & 0.9 & 0.0000 & 0.0000 & 0.0000 & 0.0000 & 0.0000 & \multirow{5}{*}{0.0000} \\
\hline & 0.92 & 0.0000 & 0.0000 & 0.0000 & 0.0000 & 0.0000 & \\
\hline & 0.95 & 0.0001 & 0.0000 & 0.0000 & 0.0000 & 0.0000 & \\
\hline & 0.97 & 0.0001 & 0.0000 & 0.0000 & 0.0000 & 0.0000 & \\
\hline & 0.99 & 0.0001 & 0.0000 & 0.0000 & 0.0000 & 0.0000 & \\
\hline \multicolumn{2}{|c|}{ Caverage } & 0.0001 & 0.0000 & 0.0000 & 0.0000 & 0.0000 & 0.0000 \\
\hline \multirow{5}{*}{-0.95} & 0.9 & 0.0002 & 0.0000 & 0.0000 & 0.0000 & 0.0001 & \multirow{5}{*}{0.0000} \\
\hline & 0.92 & 0.0000 & 0.0000 & 0.0000 & 0.0000 & 0.0000 & \\
\hline & 0.95 & 0.0000 & 0.0000 & 0.0000 & 0.0000 & 0.0000 & \\
\hline & 0.97 & 0.0001 & 0.0001 & 0.0000 & 0.0000 & 0.0001 & \\
\hline & 0.99 & 0.0000 & 0.0001 & 0.0000 & 0.0000 & 0.0000 & \\
\hline \multicolumn{2}{|c|}{ Caverage } & 0.0001 & 0.0000 & 0.0000 & 0.0000 & 0.0000 & 0.0000 \\
\hline \multirow{5}{*}{-0.97} & 0.9 & 0.0001 & 0.0000 & 0.0000 & 0.0000 & 0.0000 & \multirow{5}{*}{0.0000} \\
\hline & 0.92 & 0.0001 & 0.0001 & 0.0000 & 0.0000 & 0.0001 & \\
\hline & 0.95 & 0.0001 & 0.0000 & 0.0000 & 0.0000 & 0.0000 & \\
\hline & 0.97 & 0.0003 & 0.0001 & 0.0001 & 0.0000 & 0.0001 & \\
\hline & 0.99 & 0.0000 & 0.0000 & 0.0000 & 0.0000 & 0.0000 & \\
\hline \multicolumn{2}{|c|}{ Caverage } & 0.0001 & 0.0000 & 0.0000 & 0.0000 & 0.0000 & 0.0000 \\
\hline \multirow{5}{*}{-0.99} & 0.9 & 0.0000 & 0.0000 & 0.0000 & 0.0000 & 0.0000 & \multirow{5}{*}{0.0000} \\
\hline & 0.92 & 0.0000 & 0.0000 & 0.0000 & 0.0000 & 0.0000 & \\
\hline & 0.95 & 0.0001 & 0.0000 & 0.0000 & 0.0000 & 0.0000 & \\
\hline & 0.97 & 0.0000 & 0.0000 & 0.0000 & 0.0000 & 0.0000 & \\
\hline & 0.99 & 0.0000 & 0.0000 & 0.0000 & 0.0000 & 0.0000 & \\
\hline \multicolumn{2}{|c|}{ Caverage } & 0.0001 & 0.0000 & 0.0000 & 0.0000 & 0.0000 & 0.0000 \\
\hline
\end{tabular}

Note: 'Raverage' (stands for row average) is the average rejection rate for different values of $n$ for the same case; 'Caverage' (stands for column average) is the average rejection rate for different cases in which $\alpha_{1} \in A^{+}=$ $\{0.99,0.97,0.95,0.92,0.9\}$ for the same $n$ for the same Situation; 'Saverage' (stands for Situation average) is the average rejection rate for different cases in which $\alpha_{1} \in A^{+}=\{0.99,0.97,0.95,0.92,0.9\}$ and different $n$ for the same situation and 'overall average' in the 3 to 6 columns is the overall average for each $n$ for all situations and all cases while 'overall average' in the last two column is the overall average for all the cases in the entire table.

Table A4. Rejection Rate, Error $\sim \mathrm{t}(1)$.

\begin{tabular}{cccccccc}
\hline$\alpha_{2}$ & $\boldsymbol{\alpha}_{\mathbf{1}}$ & $\boldsymbol{n}=\mathbf{1 0 0}$ & $\boldsymbol{n}=\mathbf{2 0 0}$ & $\boldsymbol{n}=\mathbf{4 0 0}$ & $\boldsymbol{n}=\mathbf{8 0 0}$ & Raverage & Saverage \\
\hline & 0.9 & 0.0020 & 0.0005 & 0.0007 & 0.0027 & 0.0015 & \\
-0.92 & 0.0010 & 0.0006 & 0.0006 & 0.0013 & 0.0009 & \\
& 0.95 & 0.0009 & 0.0006 & 0.0002 & 0.0007 & 0.0006 & 0.0008 \\
& 0.97 & 0.0009 & 0.0007 & 0.0002 & 0.0001 & 0.0005 & \\
& 0.99 & 0.0009 & 0.0001 & 0.0000 & 0.0003 & 0.0003 & \\
\hline \multicolumn{2}{c}{ Caverage } & 0.0011 & 0.0005 & 0.0003 & 0.0010 & 0.0008 & 0.0008 \\
\hline
\end{tabular}


Table A4. Cont.

\begin{tabular}{|c|c|c|c|c|c|c|c|}
\hline$\alpha_{2}$ & $\alpha_{1}$ & $n=100$ & $n=200$ & $n=400$ & $n=800$ & Raverage & Saverage \\
\hline \multirow{5}{*}{-0.92} & 0.9 & 0.0008 & 0.0005 & 0.0002 & 0.0006 & 0.0005 & \multirow{5}{*}{0.0004} \\
\hline & 0.92 & 0.0012 & 0.0010 & 0.0002 & 0.0006 & 0.0008 & \\
\hline & 0.95 & 0.0004 & 0.0004 & 0.0003 & 0.0003 & 0.0004 & \\
\hline & 0.97 & 0.0005 & 0.0004 & 0.0000 & 0.0001 & 0.0003 & \\
\hline & 0.99 & 0.0005 & 0.0002 & 0.0001 & 0.0000 & 0.0002 & \\
\hline \multicolumn{2}{|c|}{ Caverage } & 0.0007 & 0.0005 & 0.0002 & 0.0003 & 0.0004 & 0.0004 \\
\hline \multirow{5}{*}{-0.95} & 0.9 & 0.0008 & 0.0007 & 0.0002 & 0.0005 & 0.0006 & \multirow{5}{*}{0.0004} \\
\hline & 0.92 & 0.0010 & 0.0002 & 0.0001 & 0.0003 & 0.0004 & \\
\hline & 0.95 & 0.0011 & 0.0003 & 0.0002 & 0.0001 & 0.0004 & \\
\hline & 0.97 & 0.0007 & 0.0006 & 0.0000 & 0.0001 & 0.0004 & \\
\hline & 0.99 & 0.0007 & 0.0001 & 0.0000 & 0.0001 & 0.0002 & \\
\hline \multicolumn{2}{|c|}{ Caverage } & 0.0009 & 0.0004 & 0.0001 & 0.0002 & 0.0004 & 0.0004 \\
\hline \multirow{5}{*}{-0.97} & 0.9 & 0.0008 & 0.0006 & 0.0003 & 0.0003 & 0.0005 & \multirow{5}{*}{0.0004} \\
\hline & 0.92 & 0.0009 & 0.0007 & 0.0001 & 0.0001 & 0.0005 & \\
\hline & 0.95 & 0.0014 & 0.0001 & 0.0001 & 0.0002 & 0.0005 & \\
\hline & 0.97 & 0.0002 & 0.0002 & 0.0001 & 0.0002 & 0.0002 & \\
\hline & 0.99 & 0.0010 & 0.0002 & 0.0002 & 0.0000 & 0.0004 & \\
\hline \multicolumn{2}{|c|}{ Caverage } & 0.0009 & 0.0004 & 0.0002 & 0.0002 & 0.0004 & 0.0004 \\
\hline \multirow{5}{*}{-0.99} & 0.9 & 0.0011 & 0.0003 & 0.0004 & 0.0000 & 0.0005 & \multirow{5}{*}{0.0003} \\
\hline & 0.92 & 0.0005 & 0.0002 & 0.0001 & 0.0000 & 0.0002 & \\
\hline & 0.95 & 0.0003 & 0.0003 & 0.0000 & 0.0001 & 0.0002 & \\
\hline & 0.97 & 0.0009 & 0.0003 & 0.0001 & 0.0000 & 0.0003 & \\
\hline & 0.99 & 0.0005 & 0.0000 & 0.0000 & 0.0000 & 0.0001 & \\
\hline \multicolumn{2}{|c|}{ Caverage } & 0.0007 & 0.0002 & 0.0001 & 0.0000 & 0.0003 & 0.0003 \\
\hline \multicolumn{2}{|c|}{ Oaverage } & 0.0008 & 0.0004 & 0.0002 & 0.0003 & 0.0004 & 0.0004 \\
\hline
\end{tabular}

Note: 'Raverage' (stands for row average) is the average rejection rate for different values of $n$ for the same case; 'Caverage' (stands for column average) is the average rejection rate for different cases in which $\alpha_{1} \in A^{+}=$ $\{0.99,0.97,0.95,0.92,0.9\}$ for the same $n$ for the same Situation; 'Saverage' (stands for Situation average) is the average rejection rate for different cases in which $\alpha_{1} \in A^{+}=\{0.99,0.97,0.95,0.92,0.9\}$ and different $n$ for the same situation and 'overall average' in the 3 to 6 columns is the overall average for each $n$ for all situations and all cases while 'overall average' in the last two column is the overall average for all the cases in the entire table.

Appendix B. $\left(\alpha_{1}, \alpha_{2}\right) \in\left(A^{-}, A^{+}\right)$

Table A5. Rejection rate, error $\sim \mathrm{N}(0,1)$.

\begin{tabular}{|c|c|c|c|c|c|c|c|}
\hline$\alpha_{2}$ & $\alpha_{1}$ & $n=100$ & $n=200$ & $n=400$ & $n=800$ & Raverage & Saverage \\
\hline \multirow{5}{*}{0.9} & -0.9 & 0.0000 & 0.0000 & 0.0000 & 0.0000 & 0.0000 & \multirow{5}{*}{0.0000} \\
\hline & -0.92 & 0.0000 & 0.0000 & 0.0000 & 0.0000 & 0.0000 & \\
\hline & -0.95 & 0.0000 & 0.0000 & 0.0000 & 0.0000 & 0.0000 & \\
\hline & -0.97 & 0.0000 & 0.0000 & 0.0000 & 0.0000 & 0.0000 & \\
\hline & -0.99 & 0.0000 & 0.0000 & 0.0000 & 0.0000 & 0.0000 & \\
\hline \multicolumn{2}{|c|}{ Caverage } & 0.0000 & 0.0000 & 0.0000 & 0.0000 & 0.0000 & 0.0000 \\
\hline \multirow{5}{*}{0.92} & -0.9 & 0.0000 & 0.0000 & 0.0000 & 0.0000 & 0.0000 & \multirow{5}{*}{0.0000} \\
\hline & -0.92 & 0.0000 & 0.0000 & 0.0000 & 0.0000 & 0.0000 & \\
\hline & -0.95 & 0.0000 & 0.0000 & 0.0000 & 0.0000 & 0.0000 & \\
\hline & -0.97 & 0.0000 & 0.0000 & 0.0000 & 0.0000 & 0.0000 & \\
\hline & -0.99 & 0.0000 & 0.0000 & 0.0000 & 0.0000 & 0.0000 & \\
\hline \multicolumn{2}{|c|}{ Caverage } & 0.0000 & 0.0000 & 0.0000 & 0.0000 & 0.0000 & 0.0000 \\
\hline
\end{tabular}


Table A5. Cont.

\begin{tabular}{|c|c|c|c|c|c|c|c|}
\hline$\alpha_{2}$ & $\alpha_{1}$ & $n=100$ & $n=200$ & $n=400$ & $n=800$ & Raverage & Saverage \\
\hline \multirow{5}{*}{0.95} & -0.9 & 0.0000 & 0.0000 & 0.0000 & 0.0000 & 0.0000 & \multirow{5}{*}{0.0000} \\
\hline & -0.92 & 0.0000 & 0.0000 & 0.0000 & 0.0000 & 0.0000 & \\
\hline & -0.95 & 0.0000 & 0.0000 & 0.0000 & 0.0000 & 0.0000 & \\
\hline & -0.97 & 0.0000 & 0.0000 & 0.0000 & 0.0000 & 0.0000 & \\
\hline & -0.99 & 0.0000 & 0.0000 & 0.0000 & 0.0000 & 0.0000 & \\
\hline \multicolumn{2}{|c|}{ Caverage } & 0.0000 & 0.0000 & 0.0000 & 0.0000 & 0.0000 & 0.0000 \\
\hline \multirow{5}{*}{0.97} & -0.9 & 0.0000 & 0.0000 & 0.0000 & 0.0000 & 0.0000 & \multirow{5}{*}{0.0000} \\
\hline & -0.92 & 0.0000 & 0.0000 & 0.0000 & 0.0000 & 0.0000 & \\
\hline & -0.95 & 0.0000 & 0.0000 & 0.0000 & 0.0000 & 0.0000 & \\
\hline & -0.97 & 0.0000 & 0.0000 & 0.0000 & 0.0000 & 0.0000 & \\
\hline & -0.99 & 0.0000 & 0.0000 & 0.0000 & 0.0000 & 0.0000 & \\
\hline \multicolumn{2}{|c|}{ Caverage } & 0.0000 & 0.0000 & 0.0000 & 0.0000 & 0.0000 & 0.0000 \\
\hline \multirow{5}{*}{0.99} & -0.9 & 0.0000 & 0.0000 & 0.0000 & 0.0000 & 0.0000 & \multirow{5}{*}{0.0000} \\
\hline & -0.92 & 0.0000 & 0.0000 & 0.0000 & 0.0000 & 0.0000 & \\
\hline & -0.95 & 0.0000 & 0.0000 & 0.0000 & 0.0000 & 0.0000 & \\
\hline & -0.97 & 0.0000 & 0.0000 & 0.0000 & 0.0000 & 0.0000 & \\
\hline & -0.99 & 0.0000 & 0.0000 & 0.0000 & 0.0000 & 0.0000 & \\
\hline \multicolumn{2}{|c|}{ Caverage } & 0.0000 & 0.0000 & 0.0000 & 0.0000 & 0.0000 & 0.0000 \\
\hline \multicolumn{2}{|c|}{ Oaverage } & 0.0000 & 0.0000 & 0.0000 & 0.0000 & 0.0000 & 0.0000 \\
\hline
\end{tabular}

Note: 'Raverage' (stands for row average) is the average rejection rate for different values of $n$ for the same case; 'Caverage' (stands for column average) is the average rejection rate for different cases in which $\alpha_{1} \in A^{-}=$ $\{-0.99,-0.97,-0.95,-0.92,-0.9\}$ for the same $n$ for the same Situation; 'Saverage' (stands for Situation average) is the average rejection rate for different cases in which $\alpha_{1} \in A^{-}=\{-0.99,-0.97,-0.95,-0.92,-0.9\}$ and different $n$ for the same situation and 'overall average' in the 3 to 6 columns is the overall average for each $n$ for all situations and all cases while 'overall average' in the last two column is the overall average for all the cases in the entire table.

Table A6. Rejection rate, error $\sim \mathrm{t}(5)$.

\begin{tabular}{|c|c|c|c|c|c|c|c|}
\hline$\alpha_{2}$ & $\alpha_{1}$ & $n=100$ & $n=200$ & $n=400$ & $n=800$ & Raverage & Saverage \\
\hline \multirow{5}{*}{0.9} & -0.9 & 0.0000 & 0.0000 & 0.0000 & 0.0000 & 0.0000 & \multirow{5}{*}{0.0000} \\
\hline & -0.92 & 0.0000 & 0.0000 & 0.0000 & 0.0000 & 0.0000 & \\
\hline & -0.95 & 0.0000 & 0.0000 & 0.0000 & 0.0000 & 0.0000 & \\
\hline & -0.97 & 0.0000 & 0.0000 & 0.0000 & 0.0000 & 0.0000 & \\
\hline & -0.99 & 0.0000 & 0.0000 & 0.0000 & 0.0000 & 0.0000 & \\
\hline \multicolumn{2}{|c|}{ Caverage } & 0.0000 & 0.0000 & 0.0000 & 0.0000 & 0.0000 & 0.0000 \\
\hline \multirow{5}{*}{0.92} & -0.9 & 0.0000 & 0.0000 & 0.0000 & 0.0000 & 0.0000 & \multirow{5}{*}{0.0000} \\
\hline & -0.92 & 0.0001 & 0.0000 & 0.0000 & 0.0000 & 0.0000 & \\
\hline & -0.95 & 0.0000 & 0.0000 & 0.0000 & 0.0000 & 0.0000 & \\
\hline & -0.97 & 0.0000 & 0.0000 & 0.0000 & 0.0000 & 0.0000 & \\
\hline & -0.99 & 0.0000 & 0.0000 & 0.0000 & 0.0000 & 0.0000 & \\
\hline \multicolumn{2}{|c|}{ Caverage } & 0.0000 & 0.0000 & 0.0000 & 0.0000 & 0.0000 & 0.0000 \\
\hline \multirow{5}{*}{0.95} & -0.9 & 0.0000 & 0.0000 & 0.0000 & 0.0000 & 0.0000 & \multirow{5}{*}{0.0000} \\
\hline & -0.92 & 0.0000 & 0.0000 & 0.0000 & 0.0000 & 0.0000 & \\
\hline & -0.95 & 0.0000 & 0.0000 & 0.0000 & 0.0000 & 0.0000 & \\
\hline & -0.97 & 0.0000 & 0.0000 & 0.0000 & 0.0000 & 0.0000 & \\
\hline & -0.99 & 0.0000 & 0.0000 & 0.0000 & 0.0000 & 0.0000 & \\
\hline \multicolumn{2}{|c|}{ Caverage } & 0.0000 & 0.0000 & 0.0000 & 0.0000 & 0.0000 & 0.0000 \\
\hline
\end{tabular}


Table A6. Cont.

\begin{tabular}{|c|c|c|c|c|c|c|c|}
\hline$\alpha_{2}$ & $\alpha_{1}$ & $n=100$ & $n=200$ & $n=400$ & $n=800$ & Raverage & Saverage \\
\hline \multirow{5}{*}{0.97} & -0.9 & 0.0000 & 0.0000 & 0.0000 & 0.0000 & 0.0000 & \multirow{5}{*}{0.0000} \\
\hline & -0.92 & 0.0000 & 0.0000 & 0.0000 & 0.0000 & 0.0000 & \\
\hline & -0.95 & 0.0000 & 0.0000 & 0.0000 & 0.0000 & 0.0000 & \\
\hline & -0.97 & 0.0000 & 0.0000 & 0.0000 & 0.0000 & 0.0000 & \\
\hline & -0.99 & 0.0000 & 0.0000 & 0.0000 & 0.0000 & 0.0000 & \\
\hline \multicolumn{2}{|c|}{ Caverage } & 0.0000 & 0.0000 & 0.0000 & 0.0000 & 0.0000 & 0.0000 \\
\hline \multirow{5}{*}{0.99} & -0.9 & 0.0001 & 0.0000 & 0.0000 & 0.0000 & 0.0000 & \multirow{5}{*}{0.0000} \\
\hline & -0.92 & 0.0000 & 0.0000 & 0.0000 & 0.0000 & 0.0000 & \\
\hline & -0.95 & 0.0000 & 0.0000 & 0.0000 & 0.0000 & 0.0000 & \\
\hline & -0.97 & 0.0000 & 0.0000 & 0.0000 & 0.0000 & 0.0000 & \\
\hline & -0.99 & 0.0000 & 0.0000 & 0.0000 & 0.0000 & 0.0000 & \\
\hline \multicolumn{2}{|c|}{ Caverage } & 0.0000 & 0.0000 & 0.0000 & 0.0000 & 0.0000 & 0.0000 \\
\hline \multicolumn{2}{|c|}{ Oaverage } & 0.0000 & 0.0000 & 0.0000 & 0.0000 & 0.0000 & 0.0000 \\
\hline
\end{tabular}

Note: 'Raverage' (stands for row average) is the average rejection rate for different values of $n$ for the same case; 'Caverage' (stands for column average) is the average rejection rate for different cases in which $\alpha_{1} \in A^{-}=$ $\{-0.99,-0.97,-0.95,-0.92,-0.9\}$ for the same $n$ for the same Situation; 'Saverage' (stands for Situation average) is the average rejection rate for different cases in which $\alpha_{1} \in A^{-}=\{-0.99,-0.97,-0.95,-0.92,-0.9\}$ and different $n$ for the same situation and 'overall average' in the 3 to 6 columns is the overall average for each $n$ for all situations and all cases while 'overall average' in the last two column is the overall average for all the cases in the entire table.

Table A7. Rejection rate, error $\sim \mathrm{t}(2)$.

\begin{tabular}{|c|c|c|c|c|c|c|c|}
\hline$\alpha_{2}$ & $\alpha_{1}$ & $n=100$ & $n=200$ & $n=400$ & $n=800$ & Raverage & Saverage \\
\hline \multirow{5}{*}{0.9} & -0.9 & 0.0000 & 0.0002 & 0.0000 & 0.0001 & 0.0001 & \multirow{5}{*}{0.0000} \\
\hline & -0.92 & 0.0000 & 0.0000 & 0.0000 & 0.0000 & 0.0000 & \\
\hline & -0.95 & 0.0000 & 0.0000 & 0.0000 & 0.0000 & 0.0000 & \\
\hline & -0.97 & 0.0000 & 0.0000 & 0.0000 & 0.0000 & 0.0000 & \\
\hline & -0.99 & 0.0000 & 0.0001 & 0.0000 & 0.0000 & 0.0000 & \\
\hline \multicolumn{2}{|c|}{ Caverage } & 0.0000 & 0.0001 & 0.0000 & 0.0000 & 0.0000 & 0.0000 \\
\hline \multirow{5}{*}{0.92} & -0.9 & 0.0001 & 0.0000 & 0.0000 & 0.0001 & 0.0001 & \multirow{5}{*}{0.0000} \\
\hline & -0.92 & 0.0000 & 0.0001 & 0.0000 & 0.0000 & 0.0000 & \\
\hline & -0.95 & 0.0001 & 0.0000 & 0.0000 & 0.0000 & 0.0000 & \\
\hline & -0.97 & 0.0000 & 0.0000 & 0.0000 & 0.0000 & 0.0000 & \\
\hline & -0.99 & 0.0000 & 0.0000 & 0.0000 & 0.0000 & 0.0000 & \\
\hline \multicolumn{2}{|c|}{ Caverage } & 0.0000 & 0.0000 & 0.0000 & 0.0000 & 0.0000 & 0.0000 \\
\hline \multirow{5}{*}{0.95} & -0.9 & 0.0000 & 0.0000 & 0.0000 & 0.0000 & 0.0000 & \multirow{5}{*}{0.0000} \\
\hline & -0.92 & 0.0000 & 0.0001 & 0.0000 & 0.0000 & 0.0000 & \\
\hline & -0.95 & 0.0002 & 0.0000 & 0.0000 & 0.0000 & 0.0001 & \\
\hline & -0.97 & 0.0000 & 0.0000 & 0.0000 & 0.0000 & 0.0000 & \\
\hline & -0.99 & 0.0000 & 0.0001 & 0.0000 & 0.0001 & 0.0001 & \\
\hline \multicolumn{2}{|c|}{ Caverage } & 0.0000 & 0.0000 & 0.0000 & 0.0000 & 0.0000 & 0.0000 \\
\hline \multirow{5}{*}{0.97} & -0.9 & 0.0001 & 0.0000 & 0.0000 & 0.0000 & 0.0000 & \multirow{5}{*}{0.0000} \\
\hline & -0.92 & 0.0002 & 0.0001 & 0.0000 & 0.0000 & 0.0001 & \\
\hline & -0.95 & 0.0000 & 0.0000 & 0.0000 & 0.0000 & 0.0000 & \\
\hline & -0.97 & 0.0001 & 0.0001 & 0.0000 & 0.0000 & 0.0001 & \\
\hline & -0.99 & 0.0001 & 0.0000 & 0.0000 & 0.0000 & 0.0000 & \\
\hline \multicolumn{2}{|c|}{ Caverage } & 0.0001 & 0.0000 & 0.0000 & 0.0000 & 0.0000 & 0.0000 \\
\hline
\end{tabular}


Table A7. Cont.

\begin{tabular}{cccccccc}
\hline$\alpha_{2}$ & $\alpha_{\mathbf{1}}$ & $\boldsymbol{n}=\mathbf{1 0 0}$ & $\boldsymbol{n}=\mathbf{2 0 0}$ & $\boldsymbol{n = 4 0 0}$ & $\boldsymbol{n = 8 0 0}$ & Raverage & Saverage \\
\hline & -0.9 & 0.0002 & 0.0001 & 0.0000 & 0.0000 & 0.0001 & \\
0.99 & -0.92 & 0.0001 & 0.0000 & 0.0000 & 0.0000 & 0.0000 & \\
& -0.95 & 0.0000 & 0.0000 & 0.0000 & 0.0000 & 0.0000 & 0.0000 \\
& -0.97 & 0.0000 & 0.0000 & 0.0000 & 0.0000 & 0.0000 & \\
\hline \multicolumn{2}{l}{ Caverage } & 0.0001 & 0.0000 & 0.0000 & 0.0000 & 0.0000 & 0.0000 \\
\hline \multicolumn{2}{l}{ Oaverage } & 0.0001 & 0.0000 & 0.0000 & 0.0000 & 0.0000 & \\
\hline
\end{tabular}

Note: 'Raverage' (stands for row average) is the average rejection rate for different values of $n$ for the same case; 'Caverage' (stands for column average) is the average rejection rate for different cases in which $\alpha_{1} \in A^{-}=$ $\{-0.99,-0.97,-0.95,-0.92,-0.9\}$ for the same $n$ for the same Situation; 'Saverage' (stands for Situation average) is the average rejection rate for different cases in which $\alpha_{1} \in A^{-}=\{-0.99,-0.97,-0.95,-0.92,-0.9\}$ and different $n$ for the same situation and 'overall average' in the 3 to 6 columns is the overall average for each $n$ for all situations and all cases while 'overall average' in the last two column is the overall average for all the cases in the entire table.

Table A8. Rejection rate, error $\sim \mathrm{t}(1)$.

\begin{tabular}{|c|c|c|c|c|c|c|c|}
\hline$\alpha_{2}$ & $\alpha_{1}$ & $n=100$ & $n=200$ & $n=400$ & $n=800$ & Raverage & Saverage \\
\hline \multirow{5}{*}{0.9} & -0.9 & 0.0014 & 0.0006 & 0.0009 & 0.0019 & 0.0012 & \multirow{5}{*}{0.0007} \\
\hline & -0.92 & 0.0010 & 0.0005 & 0.0004 & 0.0016 & 0.0009 & \\
\hline & -0.95 & 0.0011 & 0.0003 & 0.0004 & 0.0008 & 0.0007 & \\
\hline & -0.97 & 0.0008 & 0.0006 & 0.0000 & 0.0001 & 0.0004 & \\
\hline & -0.99 & 0.0008 & 0.0000 & 0.0001 & 0.0004 & 0.0003 & \\
\hline \multicolumn{2}{|c|}{ Caverage } & 0.0010 & 0.0004 & 0.0004 & 0.0010 & 0.0007 & 0.0007 \\
\hline \multirow{5}{*}{0.92} & -0.9 & 0.0009 & 0.0008 & 0.0002 & 0.0007 & 0.0007 & \multirow{5}{*}{0.0004} \\
\hline & -0.92 & 0.0012 & 0.0010 & 0.0002 & 0.0007 & 0.0008 & \\
\hline & -0.95 & 0.0004 & 0.0003 & 0.0003 & 0.0001 & 0.0003 & \\
\hline & -0.97 & 0.0004 & 0.0005 & 0.0000 & 0.0002 & 0.0003 & \\
\hline & -0.99 & 0.0005 & 0.0003 & 0.0001 & 0.0000 & 0.0002 & \\
\hline \multicolumn{2}{|c|}{ Caverage } & 0.0007 & 0.0006 & 0.0002 & 0.0003 & 0.0004 & 0.0004 \\
\hline \multirow{5}{*}{0.95} & -0.9 & 0.0009 & 0.0006 & 0.0002 & 0.0005 & 0.0006 & \multirow{5}{*}{0.0004} \\
\hline & -0.92 & 0.0017 & 0.0004 & 0.0002 & 0.0001 & 0.0006 & \\
\hline & -0.95 & 0.0009 & 0.0003 & 0.0003 & 0.0001 & 0.0004 & \\
\hline & -0.97 & 0.0008 & 0.0004 & 0.0001 & 0.0001 & 0.0004 & \\
\hline & -0.99 & 0.0004 & 0.0004 & 0.0000 & 0.0001 & 0.0002 & \\
\hline \multicolumn{2}{|c|}{ Caverage } & 0.0009 & 0.0004 & 0.0002 & 0.0002 & 0.0004 & 0.0004 \\
\hline \multirow{5}{*}{0.97} & -0.9 & 0.0008 & 0.0007 & 0.0005 & 0.0002 & 0.0006 & \multirow{5}{*}{0.0004} \\
\hline & -0.92 & 0.0009 & 0.0006 & 0.0000 & 0.0001 & 0.0004 & \\
\hline & -0.95 & 0.0014 & 0.0004 & 0.0002 & 0.0001 & 0.0005 & \\
\hline & -0.97 & 0.0004 & 0.0002 & 0.0000 & 0.0002 & 0.0002 & \\
\hline & -0.99 & 0.0012 & 0.0004 & 0.0001 & 0.0000 & 0.0004 & \\
\hline \multicolumn{2}{|c|}{ Caverage } & 0.0009 & 0.0005 & 0.0002 & 0.0001 & 0.0004 & 0.0004 \\
\hline
\end{tabular}


Table A8. Cont.

\begin{tabular}{cccccccc}
\hline$\alpha_{2}$ & $\boldsymbol{\alpha}_{\mathbf{1}}$ & $\boldsymbol{n}=\mathbf{1 0 0}$ & $\boldsymbol{n}=\mathbf{2 0 0}$ & $\boldsymbol{n}=\mathbf{4 0 0}$ & $\boldsymbol{n}=\mathbf{8 0 0}$ & Raverage & Saverage \\
\hline & -0.9 & 0.0010 & 0.0007 & 0.0002 & 0.0001 & 0.0005 & \\
0.99 & -0.92 & 0.0008 & 0.0006 & 0.0002 & 0.0000 & 0.0004 & \\
& -0.95 & 0.0003 & 0.0002 & 0.0003 & 0.0001 & 0.0002 & 0.0003 \\
& -0.97 & 0.0007 & 0.0004 & 0.0002 & 0.0000 & 0.0003 & \\
& -0.99 & 0.0003 & 0.0001 & 0.0002 & 0.0001 & 0.0002 & \\
\hline \multicolumn{2}{c}{ Caverage } & 0.0006 & 0.0004 & 0.0002 & 0.0001 & 0.0003 & 0.0003 \\
\hline \multicolumn{2}{c}{ Oaverage } & 0.0008 & 0.0005 & 0.0002 & 0.0003 & 0.0005 & 0.0005
\end{tabular}

Note: 'Raverage' (stands for row average) is the average rejection rate for different values of $n$ for the same case; 'Caverage' (stands for column average) is the average rejection rate for different cases in which $\alpha_{1} \in A^{-}=$ $\{-0.99,-0.97,-0.95,-0.92,-0.9\}$ for the same $n$ for the same Situation; 'Saverage' (stands for Situation average) is the average rejection rate for different cases in which $\alpha_{1} \in A^{-}=\{-0.99,-0.97,-0.95,-0.92,-0.9\}$ and different $n$ for the same situation and 'overall average' in the 3 to 6 columns is the overall average for each $n$ for all situations and all cases while 'overall average' in the last two column is the overall average for all the cases in the entire table.

Appendix C. $\left(\alpha_{1}, \alpha_{2}\right) \in\left(A^{+}, A^{+}\right)$

Table A9. Rejection rate, error $\sim \mathrm{N}(0,1)$.

\begin{tabular}{|c|c|c|c|c|c|c|c|}
\hline$\alpha_{2}$ & $\alpha_{1}$ & $n=100$ & $n=200$ & $n=400$ & $n=800$ & Raverage & Saverage \\
\hline \multirow{5}{*}{0.9} & 0.9 & 0.5105 & 0.5173 & 0.5191 & 0.5202 & 0.5168 & \multirow{5}{*}{0.5669} \\
\hline & 0.92 & 0.5128 & 0.5379 & 0.5446 & 0.5552 & 0.5376 & \\
\hline & 0.95 & 0.5528 & 0.5704 & 0.5800 & 0.5764 & 0.5699 & \\
\hline & 0.97 & 0.5640 & 0.5887 & 0.6054 & 0.6058 & 0.5910 & \\
\hline & 0.99 & 0.5847 & 0.6203 & 0.6358 & 0.6370 & 0.6195 & \\
\hline \multicolumn{2}{|c|}{ Caverage } & 0.5450 & 0.5669 & 0.5770 & 0.5789 & 0.5669 & 0.5669 \\
\hline \multirow{5}{*}{0.92} & 0.9 & 0.5193 & 0.5391 & 0.5422 & 0.5445 & 0.5363 & \multirow{5}{*}{0.5924} \\
\hline & 0.92 & 0.5392 & 0.5596 & 0.5756 & 0.5744 & 0.5622 & \\
\hline & 0.95 & 0.5633 & 0.5995 & 0.6120 & 0.6102 & 0.5963 & \\
\hline & 0.97 & 0.5880 & 0.6239 & 0.6368 & 0.6458 & 0.6236 & \\
\hline & 0.99 & 0.6028 & 0.6468 & 0.6584 & 0.6660 & 0.6435 & \\
\hline \multicolumn{2}{|c|}{ Caverage } & 0.5625 & 0.5938 & 0.6050 & 0.6082 & 0.5924 & 0.5924 \\
\hline \multirow{5}{*}{0.95} & 0.9 & 0.5451 & 0.5805 & 0.5795 & 0.5877 & 0.5732 & \multirow{5}{*}{0.6354} \\
\hline & 0.92 & 0.5660 & 0.5970 & 0.6131 & 0.6164 & 0.5981 & \\
\hline & 0.95 & 0.6120 & 0.6391 & 0.6554 & 0.6504 & 0.6392 & \\
\hline & 0.97 & 0.6252 & 0.6719 & 0.6853 & 0.6889 & 0.6678 & \\
\hline & 0.99 & 0.6486 & 0.6981 & 0.7221 & 0.7248 & 0.6984 & \\
\hline \multicolumn{2}{|c|}{ Caverage } & 0.5994 & 0.6373 & 0.6511 & 0.6536 & 0.6354 & 0.6354 \\
\hline \multirow{5}{*}{0.97} & 0.9 & 0.5591 & 0.5965 & 0.6113 & 0.6006 & 0.5919 & \multirow{5}{*}{0.6623} \\
\hline & 0.92 & 0.5833 & 0.6107 & 0.6367 & 0.6350 & 0.6164 & \\
\hline & 0.95 & 0.6282 & 0.6625 & 0.6897 & 0.6869 & 0.6668 & \\
\hline & 0.97 & 0.6433 & 0.6984 & 0.7286 & 0.7300 & 0.7001 & \\
\hline & 0.99 & 0.6767 & 0.7288 & 0.7614 & 0.7785 & 0.7364 & \\
\hline \multicolumn{2}{|c|}{ Caverage } & 0.6181 & 0.6594 & 0.6855 & 0.6862 & 0.6623 & 0.6623 \\
\hline
\end{tabular}


Table A9. Cont.

\begin{tabular}{cccccccc}
\hline$\alpha_{2}$ & $\boldsymbol{\alpha}_{\mathbf{1}}$ & $\boldsymbol{n = 1 0 0}$ & $\boldsymbol{n = 2 0 0}$ & $\boldsymbol{n}=\mathbf{4 0 0}$ & $\boldsymbol{n}=\mathbf{8 0 0}$ & Raverage & Saverage \\
\hline \multirow{4}{*}{0.99} & 0.9 & 0.5775 & 0.6051 & 0.6274 & 0.6385 & 0.6121 & \\
& 0.92 & 0.6106 & 0.6462 & 0.6625 & 0.6677 & 0.6468 & \\
& 0.95 & 0.6454 & 0.6957 & 0.7225 & 0.7240 & 0.6969 & 0.6937 \\
0.97 & 0.6669 & 0.7251 & 0.7632 & 0.7687 & 0.7310 & \\
& 0.99 & 0.7103 & 0.7730 & 0.8071 & 0.8370 & 0.7819 & \\
\hline \multicolumn{2}{c}{ Caverage } & 0.6421 & 0.6890 & 0.7165 & 0.7272 & 0.6937 & 0.6937 \\
\hline \multicolumn{2}{c}{ Oaverage } & 0.5934 & 0.6293 & 0.6470 & 0.6508 & 0.6301 & 0.6301
\end{tabular}

Note: 'Raverage' (stands for row average) is the average rejection rate for different values of $n$ for the same case; 'Caverage' (stands for column average) is the average rejection rate for different cases in which $\alpha_{1} \in A^{+}=$ $\{0.99,0.97,0.95,0.92,0.9\}$ for the same $n$ for the same Situation; 'Saverage' (stands for Situation average) is the average rejection rate for different cases in which $\alpha_{1} \in A^{+}=\{0.99,0.97,0.95,0.92,0.9\}$ and different $n$ for the same situation and 'overall average' in the 3 to 6 columns is the overall average for each $n$ for all situations and all cases while 'overall average' in the last two column is the overall average for all the cases in the entire table.

Table A10. Rejection rate, error $\sim \mathrm{t}(5)$.

\begin{tabular}{|c|c|c|c|c|c|c|c|}
\hline$\alpha_{2}$ & $\alpha_{1}$ & $n=100$ & $n=200$ & $n=400$ & $n=800$ & Raverage & Saverage \\
\hline \multirow{5}{*}{0.9} & 0.9 & 0.5104 & 0.5152 & 0.5233 & 0.5151 & 0.5160 & \multirow{5}{*}{0.5667} \\
\hline & 0.92 & 0.5228 & 0.5420 & 0.5447 & 0.5442 & 0.5384 & \\
\hline & 0.95 & 0.5514 & 0.5588 & 0.5752 & 0.5877 & 0.5683 & \\
\hline & 0.97 & 0.5608 & 0.5979 & 0.6092 & 0.6065 & 0.5936 & \\
\hline & 0.99 & 0.5805 & 0.6134 & 0.6322 & 0.6435 & 0.6174 & \\
\hline \multicolumn{2}{|c|}{ Caverage } & 0.5452 & 0.5655 & 0.5769 & 0.5794 & 0.5667 & 0.5667 \\
\hline \multirow{5}{*}{0.92} & 0.9 & 0.5207 & 0.5426 & 0.5490 & 0.5490 & 0.5403 & \multirow{5}{*}{0.5922} \\
\hline & 0.92 & 0.5384 & 0.5598 & 0.5697 & 0.5690 & 0.5592 & \\
\hline & 0.95 & 0.5757 & 0.5939 & 0.6042 & 0.6033 & 0.5943 & \\
\hline & 0.97 & 0.5834 & 0.6336 & 0.6349 & 0.6319 & 0.6210 & \\
\hline & 0.99 & 0.6102 & 0.6454 & 0.6607 & 0.6690 & 0.6463 & \\
\hline \multicolumn{2}{|c|}{ Caverage } & 0.5657 & 0.5951 & 0.6037 & 0.6044 & 0.5922 & 0.5922 \\
\hline \multirow{5}{*}{0.95} & 0.9 & 0.5539 & 0.5724 & 0.5925 & 0.5902 & 0.5773 & \multirow{5}{*}{0.6438} \\
\hline & 0.92 & 0.5729 & 0.5984 & 0.6114 & 0.6120 & 0.5987 & \\
\hline & 0.95 & 0.6088 & 0.6413 & 0.6489 & 0.6484 & 0.6369 & \\
\hline & 0.97 & 0.6198 & 0.6665 & 0.6827 & 0.6900 & 0.6648 & \\
\hline & 0.99 & 0.6427 & 0.7039 & 0.7159 & 0.7235 & 0.6965 & \\
\hline \multicolumn{2}{|c|}{ Caverage } & 0.5996 & 0.6365 & 0.6503 & 0.6528 & 0.6348 & 0.6438 \\
\hline \multirow{5}{*}{0.97} & 0.9 & 0.5723 & 0.5962 & 0.6093 & 0.6042 & 0.5955 & \multirow{5}{*}{0.6656} \\
\hline & 0.92 & 0.5950 & 0.6304 & 0.6360 & 0.6392 & 0.6252 & \\
\hline & 0.95 & 0.6291 & 0.6753 & 0.6931 & 0.6859 & 0.6709 & \\
\hline & 0.97 & 0.6448 & 0.7061 & 0.7273 & 0.7295 & 0.7019 & \\
\hline & 0.99 & 0.6669 & 0.7328 & 0.7639 & 0.7739 & 0.7344 & \\
\hline \multicolumn{2}{|c|}{ Caverage } & 0.6216 & 0.6682 & 0.6859 & 0.6865 & 0.6656 & 0.6656 \\
\hline
\end{tabular}


Table A10. Cont.

\begin{tabular}{cccccccc}
\hline$\alpha_{2}$ & $\boldsymbol{\alpha}_{\mathbf{1}}$ & $\boldsymbol{n}=\mathbf{1 0 0}$ & $\boldsymbol{n}=\mathbf{2 0 0}$ & $\boldsymbol{n}=\mathbf{4 0 0}$ & $\boldsymbol{n}=\mathbf{8 0 0}$ & Raverage & Saverage \\
\hline \multirow{4}{*}{0.99} & 0.9 & 0.5752 & 0.6114 & 0.6252 & 0.6305 & 0.6106 & \\
& 0.92 & 0.6056 & 0.6394 & 0.6601 & 0.6695 & 0.6437 & \\
& 0.95 & 0.6478 & 0.7000 & 0.7093 & 0.7203 & 0.6944 & 0.6941 \\
& 0.97 & 0.6829 & 0.7336 & 0.7657 & 0.7784 & 0.7402 & \\
& 0.99 & 0.7092 & 0.7747 & 0.8099 & 0.8340 & 0.7820 & \\
\hline \multicolumn{2}{c}{ Caverage } & 0.6441 & 0.6918 & 0.7140 & 0.7265 & 0.6941 & 0.6941 \\
\hline \multicolumn{2}{c}{ Oaverage } & 0.5952 & 0.6314 & 0.6462 & 0.6499 & 0.6307 & 0.6307
\end{tabular}

Note: 'Raverage' (stands for row average) is the average rejection rate for different values of $n$ for the same case; 'Caverage' (stands for column average) is the average rejection rate for different cases in which $\alpha_{1} \in A^{+}=$ $\{0.99,0.97,0.95,0.92,0.9\}$ for the same $n$ for the same Situation; 'Saverage' (stands for Situation average) is the average rejection rate for different cases in which $\alpha_{1} \in A^{+}=\{0.99,0.97,0.95,0.92,0.9\}$ and different $n$ for the same situation and 'overall average' in the 3 to 6 columns is the overall average for each $n$ for all situations and all cases while 'overall average' in the last two column is the overall average for all the cases in the entire table.

Table A11. Rejection rate, error $\sim \mathrm{t}(2)$.

\begin{tabular}{|c|c|c|c|c|c|c|c|}
\hline$\alpha_{2}$ & $\alpha_{1}$ & $n=100$ & $n=200$ & $n=400$ & $n=800$ & Raverage & Saverage \\
\hline \multirow{5}{*}{0.9} & 0.9 & 0.5075 & 0.5134 & 0.5077 & 0.4965 & 0.5063 & \multirow{5}{*}{0.5631} \\
\hline & 0.92 & 0.5198 & 0.5290 & 0.5310 & 0.5337 & 0.5284 & \\
\hline & 0.95 & 0.5502 & 0.5810 & 0.5791 & 0.5798 & 0.5725 & \\
\hline & 0.97 & 0.5705 & 0.5941 & 0.6000 & 0.5999 & 0.5911 & \\
\hline & 0.99 & 0.5809 & 0.6237 & 0.6312 & 0.6338 & 0.6174 & \\
\hline \multicolumn{2}{|c|}{ Caverage } & 0.5458 & 0.5682 & 0.5698 & 0.5687 & 0.5631 & 0.5631 \\
\hline \multirow{5}{*}{0.92} & 0.9 & 0.5160 & 0.5321 & 0.5458 & 0.5314 & 0.5313 & \multirow{5}{*}{0.5894} \\
\hline & 0.92 & 0.5414 & 0.5557 & 0.5604 & 0.5591 & 0.5542 & \\
\hline & 0.95 & 0.5730 & 0.5941 & 0.6058 & 0.6005 & 0.5934 & \\
\hline & 0.97 & 0.5976 & 0.6195 & 0.6263 & 0.6381 & 0.6204 & \\
\hline & 0.99 & 0.6202 & 0.6460 & 0.6618 & 0.6627 & 0.6477 & \\
\hline \multicolumn{2}{|c|}{ Caverage } & 0.5696 & 0.5895 & 0.6000 & 0.5984 & 0.5894 & 0.5894 \\
\hline \multirow{5}{*}{0.95} & 0.9 & 0.5593 & 0.5797 & 0.5696 & 0.5736 & 0.5706 & \multirow{5}{*}{0.6341} \\
\hline & 0.92 & 0.5831 & 0.5927 & 0.5959 & 0.5982 & 0.5925 & \\
\hline & 0.95 & 0.6064 & 0.6430 & 0.6475 & 0.6449 & 0.6355 & \\
\hline & 0.97 & 0.6354 & 0.6709 & 0.6858 & 0.6882 & 0.6701 & \\
\hline & 0.99 & 0.6512 & 0.7045 & 0.7205 & 0.7321 & 0.7021 & \\
\hline \multicolumn{2}{|c|}{ Caverage } & 0.6071 & 0.6382 & 0.6439 & 0.6474 & 0.6341 & 0.6341 \\
\hline \multirow{5}{*}{0.97} & 0.9 & 0.5736 & 0.5961 & 0.5993 & 0.6070 & 0.5940 & \multirow{5}{*}{0.6660} \\
\hline & 0.92 & 0.5908 & 0.6260 & 0.6375 & 0.6308 & 0.6213 & \\
\hline & 0.95 & 0.6407 & 0.6650 & 0.6868 & 0.6908 & 0.6708 & \\
\hline & 0.97 & 0.6613 & 0.7048 & 0.7284 & 0.7340 & 0.7071 & \\
\hline & 0.99 & 0.6761 & 0.7392 & 0.7588 & 0.7735 & 0.7369 & \\
\hline \multicolumn{2}{|c|}{ Caverage } & 0.6285 & 0.6662 & 0.6822 & 0.6872 & 0.6660 & 0.6660 \\
\hline
\end{tabular}


Table A11. Cont.

\begin{tabular}{cccccccc}
\hline$\alpha_{2}$ & $\alpha_{\mathbf{1}}$ & $\boldsymbol{n}=\mathbf{1 0 0}$ & $\boldsymbol{n}=\mathbf{2 0 0}$ & $\boldsymbol{n}=\mathbf{4 0 0}$ & $\boldsymbol{n}=\mathbf{8 0 0}$ & Raverage & Saverage \\
\hline \multirow{4}{*}{0.99} & 0.9 & 0.5866 & 0.6290 & 0.6315 & 0.6386 & 0.6214 & \\
& 0.92 & 0.6175 & 0.6508 & 0.6665 & 0.6676 & 0.6506 & \\
& 0.95 & 0.6494 & 0.6986 & 0.7236 & 0.7324 & 0.7010 & 0.6997 \\
& 0.97 & 0.6891 & 0.7337 & 0.7666 & 0.7798 & 0.7423 & \\
& 0.99 & 0.7113 & 0.7687 & 0.8146 & 0.8380 & 0.7832 & \\
\hline \multicolumn{2}{c}{ Caverage } & 0.6508 & 0.6962 & 0.7206 & 0.7313 & 0.6997 & 0.6997 \\
\hline \multicolumn{2}{c}{ Oaverage } & 0.6004 & 0.6317 & 0.6433 & 0.6466 & 0.6305 & 0.6305
\end{tabular}

Note: 'Raverage' (stands for row average) is the average rejection rate for different values of $n$ for the same case; 'Caverage' (stands for column average) is the average rejection rate for different cases in which $\alpha_{1} \in A^{+}=$ $\{0.99,0.97,0.95,0.92,0.9\}$ for the same $n$ for the same Situation; 'Saverage' (stands for Situation average) is the average rejection rate for different cases in which $\alpha_{1} \in A^{+}=\{0.99,0.97,0.95,0.92,0.9\}$ and different $n$ for the same situation and 'overall average' in the 3 to 6 columns is the overall average for each $n$ for all situations and all cases while 'overall average' in the last two column is the overall average for all the cases in the entire table.

Table A12. Rejection rate, error $\sim \mathrm{t}(1)$.

\begin{tabular}{|c|c|c|c|c|c|c|c|}
\hline$\alpha_{2}$ & $\alpha_{1}$ & $n=100$ & $n=200$ & $n=400$ & $n=800$ & Raverage & Saverage \\
\hline \multirow{5}{*}{0.9} & 0.9 & 0.4804 & 0.4400 & 0.3874 & 0.3251 & 0.4082 & \multirow{5}{*}{0.5000} \\
\hline & 0.92 & 0.5024 & 0.4703 & 0.4126 & 0.3588 & 0.4360 & \\
\hline & 0.95 & 0.5545 & 0.5287 & 0.4806 & 0.4259 & 0.4974 & \\
\hline & 0.97 & 0.5883 & 0.5764 & 0.5489 & 0.4802 & 0.5485 & \\
\hline & 0.99 & 0.6014 & 0.6250 & 0.6280 & 0.5858 & 0.6101 & \\
\hline \multicolumn{2}{|c|}{ Caverage } & 0.5454 & 0.5281 & 0.4915 & 0.4352 & 0.5000 & 0.5000 \\
\hline \multirow{5}{*}{0.92} & 0.9 & 0.5148 & 0.4843 & 0.4174 & 0.3511 & 0.4419 & \multirow{5}{*}{0.5344} \\
\hline & 0.92 & 0.5403 & 0.5129 & 0.4579 & 0.3836 & 0.4737 & \\
\hline & 0.95 & 0.5703 & 0.5592 & 0.5233 & 0.4588 & 0.5279 & \\
\hline & 0.97 & 0.6072 & 0.6253 & 0.5828 & 0.5254 & 0.5852 & \\
\hline & 0.99 & 0.6256 & 0.6597 & 0.6602 & 0.6281 & 0.6434 & \\
\hline \multicolumn{2}{|c|}{ Caverage } & 0.5716 & 0.5683 & 0.5283 & 0.4694 & 0.5344 & 0.5344 \\
\hline \multirow{5}{*}{0.95} & 0.9 & 0.5466 & 0.5294 & 0.4787 & 0.4128 & 0.4919 & \multirow{5}{*}{0.5906} \\
\hline & 0.92 & 0.5742 & 0.5762 & 0.5168 & 0.4573 & 0.5311 & \\
\hline & 0.95 & 0.6191 & 0.6213 & 0.5884 & 0.5311 & 0.5900 & \\
\hline & 0.97 & 0.6406 & 0.6638 & 0.6519 & 0.5984 & 0.6387 & \\
\hline & 0.99 & 0.6572 & 0.7063 & 0.7291 & 0.7128 & 0.7014 & \\
\hline \multicolumn{2}{|c|}{ Caverage } & 0.6075 & 0.6194 & 0.5930 & 0.5425 & 0.5906 & 0.5906 \\
\hline \multirow{5}{*}{0.97} & 0.9 & 0.5802 & 0.5792 & 0.5415 & 0.4867 & 0.5469 & \multirow{5}{*}{0.6401} \\
\hline & 0.92 & 0.6042 & 0.6231 & 0.5853 & 0.5240 & 0.5842 & \\
\hline & 0.95 & 0.6435 & 0.6701 & 0.6516 & 0.6039 & 0.6423 & \\
\hline & 0.97 & 0.6524 & 0.7112 & 0.7070 & 0.6761 & 0.6867 & \\
\hline & 0.99 & 0.6907 & 0.7415 & 0.7681 & 0.7625 & 0.7407 & \\
\hline \multicolumn{2}{|c|}{ Caverage } & 0.6342 & 0.6650 & 0.6507 & 0.6106 & 0.6401 & 0.6401 \\
\hline
\end{tabular}


Table A12. Cont.

\begin{tabular}{cccccccc}
\hline$\alpha_{2}$ & $\boldsymbol{\alpha}_{\mathbf{1}}$ & $\boldsymbol{n}=\mathbf{1 0 0}$ & $\boldsymbol{n}=\mathbf{2 0 0}$ & $\boldsymbol{n}=\mathbf{4 0 0}$ & $\boldsymbol{n}=\mathbf{8 0 0}$ & Raverage & Saverage \\
\hline \multirow{4}{*}{0.99} & 0.9 & 0.6017 & 0.6346 & 0.6252 & 0.6024 & 0.6160 & \\
& 0.92 & 0.6282 & 0.6511 & 0.6643 & 0.6414 & 0.6463 & \\
& 0.95 & 0.6562 & 0.6983 & 0.7158 & 0.6990 & 0.6923 & 0.6951 \\
& 0.97 & 0.6832 & 0.7425 & 0.7651 & 0.7607 & 0.7379 & \\
& 0.99 & 0.7150 & 0.7673 & 0.8213 & 0.8295 & 0.7833 & \\
\hline \multicolumn{2}{c}{ Caverage } & 0.6569 & 0.6988 & 0.7183 & 0.7066 & 0.6951 & 0.6951 \\
\hline \multicolumn{2}{c}{ Oaverage } & 0.6031 & 0.6159 & 0.5964 & 0.5529 & 0.5921 & 0.5921
\end{tabular}

Note: 'Raverage' (stands for row average) is the average rejection rate for different values of $n$ for the same case; 'Caverage' (stands for column average) is the average rejection rate for different cases in which $\alpha_{1} \in A^{+}=$ $\{0.99,0.97,0.95,0.92,0.9\}$ for the same $n$ for the same Situation; 'Saverage' (stands for Situation average) is the average rejection rate for different cases in which $\alpha_{1} \in A^{+}=\{0.99,0.97,0.95,0.92,0.9\}$ and different $n$ for the same situation and 'overall average' in the 3 to 6 columns is the overall average for each $n$ for all situations and all cases while 'overall average' in the last two column is the overall average for all the cases in the entire table.

Appendix D. $\left(\alpha_{1}, \alpha_{2}\right) \in\left(A^{-}, A^{-}\right)$

Table A13. Rejection rate, error $\sim \mathrm{N}(0,1)$.

\begin{tabular}{|c|c|c|c|c|c|c|c|}
\hline$\alpha_{2}$ & $\alpha_{1}$ & $n=100$ & $n=200$ & $n=400$ & $n=800$ & Raverage & Saverage \\
\hline \multirow{5}{*}{-0.9} & -0.9 & 0.5173 & 0.5196 & 0.5228 & 0.5219 & 0.5204 & \multirow{5}{*}{0.5761} \\
\hline & -0.92 & 0.5413 & 0.5469 & 0.5444 & 0.5419 & 0.5436 & \\
\hline & -0.95 & 0.5759 & 0.5852 & 0.5860 & 0.5783 & 0.5814 & \\
\hline & -0.97 & 0.6010 & 0.6006 & 0.5998 & 0.6084 & 0.6025 & \\
\hline & -0.99 & 0.6234 & 0.6360 & 0.6361 & 0.6348 & 0.6326 & \\
\hline \multicolumn{2}{|c|}{ Caverage } & 0.5718 & 0.5777 & 0.5778 & 0.5771 & 0.5761 & 0.5761 \\
\hline \multirow{5}{*}{-0.92} & -0.9 & 0.5407 & 0.5405 & 0.5435 & 0.5434 & 0.5420 & \multirow{5}{*}{0.6034} \\
\hline & -0.92 & 0.5637 & 0.5691 & 0.5687 & 0.5730 & 0.5686 & \\
\hline & -0.95 & 0.5945 & 0.6085 & 0.6101 & 0.6099 & 0.6058 & \\
\hline & -0.97 & 0.6255 & 0.6345 & 0.6330 & 0.6388 & 0.6330 & \\
\hline & -0.99 & 0.6600 & 0.6635 & 0.6746 & 0.6724 & 0.6676 & \\
\hline \multicolumn{2}{|c|}{ Caverage } & 0.5969 & 0.6032 & 0.6060 & 0.6075 & 0.6034 & 0.6034 \\
\hline \multirow{5}{*}{-0.95} & -0.9 & 0.5701 & 0.5755 & 0.5811 & 0.5755 & 0.5756 & \multirow{5}{*}{0.6478} \\
\hline & -0.92 & 0.5948 & 0.6122 & 0.6034 & 0.6077 & 0.6045 & \\
\hline & -0.95 & 0.6445 & 0.6582 & 0.6578 & 0.6568 & 0.6543 & \\
\hline & -0.97 & 0.6730 & 0.6868 & 0.6909 & 0.6867 & 0.6844 & \\
\hline & -0.99 & 0.7085 & 0.7217 & 0.7239 & 0.7267 & 0.7202 & \\
\hline \multicolumn{2}{|c|}{ Caverage } & 0.6382 & 0.6509 & 0.6514 & 0.6507 & 0.6478 & 0.6478 \\
\hline \multirow{5}{*}{-0.97} & -0.9 & 0.6038 & 0.6032 & 0.6149 & 0.6107 & 0.6082 & \multirow{5}{*}{0.6868} \\
\hline & -0.92 & 0.6284 & 0.6394 & 0.6403 & 0.6524 & 0.6401 & \\
\hline & -0.95 & 0.6850 & 0.6862 & 0.6896 & 0.6939 & 0.6887 & \\
\hline & -0.97 & 0.7172 & 0.7305 & 0.7268 & 0.7274 & 0.7255 & \\
\hline & -0.99 & 0.7546 & 0.7662 & 0.7781 & 0.7866 & 0.7714 & \\
\hline \multicolumn{2}{|c|}{ Caverage } & 0.6778 & 0.6851 & 0.6899 & 0.6942 & 0.6868 & 0.6868 \\
\hline
\end{tabular}


Table A13. Cont.

\begin{tabular}{|c|c|c|c|c|c|c|c|}
\hline$\alpha_{2}$ & $\alpha_{1}$ & $n=100$ & $n=200$ & $n=400$ & $n=800$ & Raverage & Saverage \\
\hline \multirow{5}{*}{-0.99} & -0.9 & 0.6254 & 0.6229 & 0.6409 & 0.6404 & 0.6324 & \multirow{5}{*}{0.7237} \\
\hline & -0.92 & 0.6588 & 0.6607 & 0.6595 & 0.6741 & 0.6633 & \\
\hline & -0.95 & 0.7059 & 0.7216 & 0.7328 & 0.7297 & 0.7225 & \\
\hline & -0.97 & 0.7521 & 0.7657 & 0.7790 & 0.7867 & 0.7709 & \\
\hline & -0.99 & 0.8010 & 0.8328 & 0.8365 & 0.8480 & 0.8296 & \\
\hline \multicolumn{2}{|c|}{ Caverage } & 0.7086 & 0.7207 & 0.7297 & 0.7358 & 0.7237 & 0.7237 \\
\hline \multicolumn{2}{|c|}{ Oaverage } & 0.6387 & 0.6475 & 0.6510 & 0.6530 & 0.6476 & 0.6476 \\
\hline
\end{tabular}

Note: 'Raverage' (stands for row average) is the average rejection rate for different values of $n$ for the same case; 'Caverage' (stands for column average) is the average rejection rate for different cases in which $\alpha_{1} \in A^{-}=$ $\{-0.99,-0.97,-0.95,-0.92,-0.9\}$ for the same $n$ for the same Situation; 'Saverage' (stands for Situation average) is the average rejection rate for different cases in which $\alpha_{1} \in A^{-}=\{-0.99,-0.97,-0.95,-0.92,-0.9\}$ and different $n$ for the same situation and 'overall average' in the 3 to 6 columns is the overall average for each $n$ for all situations and all cases while 'overall average' in the last two column is the overall average for all the cases in the entire table.

Table A14. Rejection rate, error $\sim \mathrm{t}(5)$.

\begin{tabular}{|c|c|c|c|c|c|c|c|}
\hline$\alpha_{2}$ & $\alpha_{1}$ & $n=100$ & $n=200$ & $n=400$ & $n=800$ & Raverage & Saverage \\
\hline \multirow{5}{*}{-0.9} & -0.9 & 0.5225 & 0.5248 & 0.5206 & 0.5201 & 0.5220 & \multirow{5}{*}{0.5783} \\
\hline & -0.92 & 0.5422 & 0.5476 & 0.5407 & 0.5536 & 0.5460 & \\
\hline & -0.95 & 0.5778 & 0.5881 & 0.5766 & 0.5753 & 0.5795 & \\
\hline & -0.97 & 0.6067 & 0.6046 & 0.6102 & 0.6062 & 0.6069 & \\
\hline & -0.99 & 0.6292 & 0.6359 & 0.6362 & 0.6473 & 0.6372 & \\
\hline \multicolumn{2}{|c|}{ Caverage } & 0.5757 & 0.5802 & 0.5769 & 0.5805 & 0.5783 & 0.5783 \\
\hline \multirow{5}{*}{-0.92} & -0.9 & 0.5404 & 0.5435 & 0.5434 & 0.5410 & 0.5421 & \multirow{5}{*}{0.6045} \\
\hline & -0.92 & 0.5674 & 0.5709 & 0.5717 & 0.5793 & 0.5723 & \\
\hline & -0.95 & 0.5939 & 0.5993 & 0.5988 & 0.6146 & 0.6017 & \\
\hline & -0.97 & 0.6354 & 0.6446 & 0.6352 & 0.6476 & 0.6407 & \\
\hline & -0.99 & 0.6550 & 0.6670 & 0.6704 & 0.6695 & 0.6655 & \\
\hline \multicolumn{2}{|c|}{ Caverage } & 0.5984 & 0.6051 & 0.6039 & 0.6104 & 0.6045 & 0.6045 \\
\hline \multirow{5}{*}{-0.95} & -0.9 & 0.5722 & 0.5734 & 0.5852 & 0.5819 & 0.5782 & \multirow{5}{*}{0.6501} \\
\hline & -0.92 & 0.6048 & 0.6094 & 0.6097 & 0.6123 & 0.6091 & \\
\hline & -0.95 & 0.6434 & 0.6499 & 0.6598 & 0.6439 & 0.6493 & \\
\hline & -0.97 & 0.6841 & 0.6888 & 0.6933 & 0.6898 & 0.6890 & \\
\hline & -0.99 & 0.7103 & 0.7263 & 0.7365 & 0.7265 & 0.7249 & \\
\hline \multicolumn{2}{|c|}{ Caverage } & 0.6430 & 0.6496 & 0.6569 & 0.6509 & 0.6501 & 0.6501 \\
\hline \multirow{5}{*}{-0.97} & -0.9 & 0.6033 & 0.6025 & 0.6084 & 0.6087 & 0.6057 & \multirow{5}{*}{0.6845} \\
\hline & -0.92 & 0.6363 & 0.6247 & 0.6339 & 0.6396 & 0.6336 & \\
\hline & -0.95 & 0.6801 & 0.6914 & 0.6917 & 0.6916 & 0.6887 & \\
\hline & -0.97 & 0.7161 & 0.7273 & 0.7294 & 0.7295 & 0.7256 & \\
\hline & -0.99 & 0.7502 & 0.7691 & 0.7751 & 0.7811 & 0.7689 & \\
\hline \multicolumn{2}{|c|}{ Caverage } & 0.6772 & 0.6830 & 0.6877 & 0.6901 & 0.6845 & 0.6845 \\
\hline
\end{tabular}


Table A14. Cont.

\begin{tabular}{|c|c|c|c|c|c|c|c|}
\hline$\alpha_{2}$ & $\alpha_{1}$ & $n=100$ & $n=200$ & $n=400$ & $n=800$ & Raverage & Saverage \\
\hline \multirow{5}{*}{-0.99} & -0.9 & 0.6334 & 0.6324 & 0.6417 & 0.6349 & 0.6356 & \multirow{5}{*}{0.7248} \\
\hline & -0.92 & 0.6624 & 0.6638 & 0.6688 & 0.6720 & 0.6668 & \\
\hline & -0.95 & 0.7057 & 0.7218 & 0.7296 & 0.7299 & 0.7218 & \\
\hline & -0.97 & 0.7579 & 0.7688 & 0.7798 & 0.7776 & 0.7710 & \\
\hline & -0.99 & 0.7967 & 0.8320 & 0.8402 & 0.8457 & 0.8287 & \\
\hline \multicolumn{2}{|c|}{ Caverage } & 0.7112 & 0.7238 & 0.7320 & 0.7320 & 0.7248 & 0.7248 \\
\hline \multicolumn{2}{|c|}{ Oaverage } & 0.6411 & 0.6483 & 0.6515 & 0.6528 & 0.6484 & 0.6484 \\
\hline
\end{tabular}

Note: 'Raverage' (stands for row average) is the average rejection rate for different values of $n$ for the same case; 'Caverage' (stands for column average) is the average rejection rate for different cases in which $\alpha_{1} \in A^{-}=$ $\{-0.99,-0.97,-0.95,-0.92,-0.9\}$ for the same $n$ for the same Situation; 'Saverage' (stands for Situation average) is the average rejection rate for different cases in which $\alpha_{1} \in A^{-}=\{-0.99,-0.97,-0.95,-0.92,-0.9\}$ and different $n$ for the same situation and 'overall average' in the 3 to 6 columns is the overall average for each $n$ for all situations and all cases while 'overall average' in the last two column is the overall average for all the cases in the entire table.

Table A15. Rejection rate, error $\sim \mathrm{t}(2)$.

\begin{tabular}{|c|c|c|c|c|c|c|c|}
\hline$\alpha_{2}$ & $\alpha_{1}$ & $n=100$ & $n=200$ & $n=400$ & $n=800$ & Raverage & Saverage \\
\hline \multirow{5}{*}{-0.9} & -0.9 & 0.5084 & 0.5231 & 0.5100 & 0.5033 & 0.5112 & \multirow{5}{*}{0.5721} \\
\hline & -0.92 & 0.5475 & 0.5319 & 0.5350 & 0.5316 & 0.5365 & \\
\hline & -0.95 & 0.5780 & 0.5737 & 0.5793 & 0.5691 & 0.5750 & \\
\hline & -0.97 & 0.6037 & 0.6119 & 0.5992 & 0.5984 & 0.6033 & \\
\hline & -0.99 & 0.6182 & 0.6434 & 0.6406 & 0.6354 & 0.6344 & \\
\hline \multicolumn{2}{|c|}{ Caverage } & 0.5712 & 0.5768 & 0.5728 & 0.5676 & 0.5721 & 0.5721 \\
\hline \multirow{5}{*}{-0.92} & -0.9 & 0.5371 & 0.5320 & 0.5341 & 0.5235 & 0.5317 & \multirow{5}{*}{0.6009} \\
\hline & -0.92 & 0.5705 & 0.5649 & 0.5668 & 0.5458 & 0.5620 & \\
\hline & -0.95 & 0.6131 & 0.5970 & 0.6031 & 0.5987 & 0.6030 & \\
\hline & -0.97 & 0.6299 & 0.6436 & 0.6354 & 0.6268 & 0.6339 & \\
\hline & -0.99 & 0.6733 & 0.6692 & 0.6711 & 0.6814 & 0.6738 & \\
\hline \multicolumn{2}{|c|}{ Caverage } & 0.6048 & 0.6013 & 0.6021 & 0.5952 & 0.6009 & 0.6009 \\
\hline \multirow{5}{*}{-0.95} & -0.9 & 0.5774 & 0.5781 & 0.5704 & 0.5729 & 0.5747 & \multirow{5}{*}{0.6495} \\
\hline & -0.92 & 0.5983 & 0.6062 & 0.5938 & 0.6024 & 0.6002 & \\
\hline & -0.95 & 0.6520 & 0.6551 & 0.6588 & 0.6521 & 0.6545 & \\
\hline & -0.97 & 0.6840 & 0.6888 & 0.6917 & 0.6902 & 0.6887 & \\
\hline & -0.99 & 0.7219 & 0.7321 & 0.7377 & 0.7270 & 0.7297 & \\
\hline \multicolumn{2}{|c|}{ Caverage } & 0.6467 & 0.6521 & 0.6505 & 0.6489 & 0.6495 & 0.6495 \\
\hline \multirow{5}{*}{-0.97} & -0.9 & 0.5938 & 0.6001 & 0.6034 & 0.5963 & 0.5984 & \multirow{5}{*}{0.6846} \\
\hline & -0.92 & 0.6337 & 0.6396 & 0.6328 & 0.6212 & 0.6318 & \\
\hline & -0.95 & 0.6930 & 0.6871 & 0.6899 & 0.6873 & 0.6893 & \\
\hline & -0.97 & 0.7265 & 0.7346 & 0.7349 & 0.7237 & 0.7299 & \\
\hline & -0.99 & 0.7635 & 0.7739 & 0.7808 & 0.7757 & 0.7735 & \\
\hline \multicolumn{2}{|c|}{ Caverage } & 0.6821 & 0.6871 & 0.6884 & 0.6808 & 0.6846 & 0.6846 \\
\hline
\end{tabular}


Table A15. Cont.

\begin{tabular}{|c|c|c|c|c|c|c|c|}
\hline$\alpha_{2}$ & $\alpha_{1}$ & $n=100$ & $n=200$ & $n=400$ & $n=800$ & Raverage & Saverage \\
\hline \multirow{5}{*}{-0.99} & -0.9 & 0.6359 & 0.6391 & 0.6361 & 0.6334 & 0.6361 & \multirow{5}{*}{0.7268} \\
\hline & -0.92 & 0.6581 & 0.6756 & 0.6675 & 0.6729 & 0.6685 & \\
\hline & -0.95 & 0.7182 & 0.7309 & 0.7299 & 0.7198 & 0.7247 & \\
\hline & -0.97 & 0.7606 & 0.7710 & 0.7822 & 0.7704 & 0.7711 & \\
\hline & -0.99 & 0.8147 & 0.8337 & 0.8424 & 0.8428 & 0.8334 & \\
\hline \multicolumn{2}{|c|}{ Caverage } & 0.7175 & 0.7301 & 0.7316 & 0.7279 & 0.7268 & 0.7268 \\
\hline \multicolumn{2}{|c|}{ Oaverage } & 0.6445 & 0.6495 & 0.6491 & 0.6441 & 0.6468 & 0.6468 \\
\hline
\end{tabular}

Note: 'Raverage' (stands for row average) is the average rejection rate for different values of $n$ for the same case; 'Caverage' (stands for column average) is the average rejection rate for different cases in which $\alpha_{1} \in A^{-}=$ $\{-0.99,-0.97,-0.95,-0.92,-0.9\}$ for the same $n$ for the same Situation; 'Saverage' (stands for Situation average) is the average rejection rate for different cases in which $\alpha_{1} \in A^{-}=\{-0.99,-0.97,-0.95,-0.92,-0.9\}$ and different $n$ for the same situation and 'overall average' in the 3 to 6 columns is the overall average for each $n$ for all situations and all cases while 'overall average' in the last two column is the overall average for all the cases in the entire table.

Table A16. Rejection rate, error $\sim \mathrm{t}(1)$.

\begin{tabular}{|c|c|c|c|c|c|c|c|}
\hline$\alpha_{2}$ & $\alpha_{1}$ & $n=100$ & $n=200$ & $n=400$ & $n=800$ & Raverage & Saverage \\
\hline \multirow{5}{*}{-0.9} & -0.9 & 0.4695 & 0.4271 & 0.3674 & 0.3162 & 0.3951 & \multirow{5}{*}{0.4882} \\
\hline & -0.92 & 0.4981 & 0.4494 & 0.3963 & 0.3422 & 0.4215 & \\
\hline & -0.95 & 0.5477 & 0.5158 & 0.4538 & 0.4070 & 0.4811 & \\
\hline & -0.97 & 0.6006 & 0.5626 & 0.5176 & 0.4592 & 0.5350 & \\
\hline & -0.99 & 0.6476 & 0.6277 & 0.5966 & 0.5613 & 0.6083 & \\
\hline \multicolumn{2}{|c|}{ Caverage } & 0.5527 & 0.5165 & 0.4663 & 0.4172 & 0.4882 & 0.4882 \\
\hline \multirow{5}{*}{-0.92} & -0.9 & 0.4982 & 0.4573 & 0.4000 & 0.3320 & 0.4219 & \multirow{5}{*}{0.5174} \\
\hline & -0.92 & 0.5349 & 0.4850 & 0.4300 & 0.3641 & 0.4535 & \\
\hline & -0.95 & 0.5857 & 0.5357 & 0.4922 & 0.4330 & 0.5117 & \\
\hline & -0.97 & 0.6268 & 0.5963 & 0.5419 & 0.4870 & 0.5630 & \\
\hline & -0.99 & 0.6642 & 0.6678 & 0.6297 & 0.5857 & 0.6369 & \\
\hline \multicolumn{2}{|c|}{ Caverage } & 0.5820 & 0.5484 & 0.4988 & 0.4404 & 0.5174 & 0.5174 \\
\hline \multirow{5}{*}{-0.95} & -0.9 & 0.5549 & 0.5045 & 0.4531 & 0.3936 & 0.4765 & \multirow{5}{*}{0.5802} \\
\hline & -0.92 & 0.5894 & 0.5474 & 0.4904 & 0.4384 & 0.5164 & \\
\hline & -0.95 & 0.6371 & 0.6096 & 0.5487 & 0.5007 & 0.5740 & \\
\hline & -0.97 & 0.6833 & 0.6588 & 0.6224 & 0.5470 & 0.6279 & \\
\hline & -0.99 & 0.7314 & 0.7280 & 0.7055 & 0.6605 & 0.7064 & \\
\hline \multicolumn{2}{|c|}{ Caverage } & 0.6392 & 0.6097 & 0.5640 & 0.5080 & 0.5802 & 0.5802 \\
\hline \multirow{5}{*}{-0.97} & -0.9 & 0.5982 & 0.5616 & 0.5063 & 0.4546 & 0.5302 & \multirow{5}{*}{0.6343} \\
\hline & -0.92 & 0.6262 & 0.5916 & 0.5457 & 0.4965 & 0.5650 & \\
\hline & -0.95 & 0.6867 & 0.6579 & 0.6199 & 0.5563 & 0.6302 & \\
\hline & -0.97 & 0.7389 & 0.7106 & 0.6681 & 0.6232 & 0.6852 & \\
\hline & -0.99 & 0.7851 & 0.7794 & 0.7557 & 0.7235 & 0.7609 & \\
\hline \multicolumn{2}{|c|}{ Caverage } & 0.6870 & 0.6602 & 0.6191 & 0.5708 & 0.6343 & 0.6343 \\
\hline
\end{tabular}


Table A16. Cont.

\begin{tabular}{cccccccc}
\hline$\alpha_{2}$ & $\alpha_{\mathbf{1}}$ & $\boldsymbol{n}=\mathbf{1 0 0}$ & $\boldsymbol{n}=\mathbf{2 0 0}$ & $\boldsymbol{n = 4 0 0}$ & $\boldsymbol{n = 8 0 0}$ & Raverage & Saverage \\
\hline & -0.9 & 0.6437 & 0.6386 & 0.5923 & 0.5597 & 0.6086 & \\
-0.99 & 0.6721 & 0.6643 & 0.6393 & 0.6028 & 0.6446 & \\
& -0.95 & 0.7295 & 0.7323 & 0.7047 & 0.6644 & 0.7077 & 0.7121 \\
& -0.97 & 0.7847 & 0.7797 & 0.7563 & 0.7301 & 0.7627 & \\
& -0.99 & 0.8333 & 0.8511 & 0.8384 & 0.8246 & 0.8369 & \\
\hline \multicolumn{2}{c}{ Caverage } & 0.7327 & 0.7332 & 0.7062 & 0.6763 & 0.7121 & 0.7121 \\
\hline \multicolumn{2}{c}{ Oaverage } & 0.6387 & 0.6136 & 0.5709 & 0.5225 & 0.5864 & 0.5864
\end{tabular}

Note: 'Raverage' (stands for row average) is the average rejection rate for different values of $n$ for the same case; 'Caverage' (stands for column average) is the average rejection rate for different cases in which $\alpha_{1} \in A^{-}=$ $\{-0.99,-0.97,-0.95,-0.92,-0.9\}$ for the same $n$ for the same Situation; 'Saverage' (stands for Situation average) is the average rejection rate for different cases in which $\alpha_{1} \in A^{-}=\{-0.99,-0.97,-0.95,-0.92,-0.9\}$ and different $n$ for the same situation and 'overall average' in the 3 to 6 columns is the overall average for each $n$ for all situations and all cases while 'overall average' in the last two column is the overall average for all the cases in the entire table.

\section{Notes}

1 We would like to thank the anonymous referee for introducing the notations of $A^{+}$and $A^{-}$.

2 We thank the anonymous referee for providing us this information.

3 We would like to thank the anonymous referee for giving us this information.

4 We would like to thank the anonymous referee for giving us this information.

\section{References}

del Barrio Castro, Tomás, Gianluca Cubadda, and Denise R. Osborn. 2020. On cointegration for processes integrated at different frequencies. In CEIS Working Paper No. 502. Glasgow: CEIS.

del Barrio Castro, Tomás, Denise R. Osborn, and A. M. Robert Taylor. 2012. On Augmented Hegy Tests For Seasonal Unit Roots. Econometric Theory 28: 1121-43. [CrossRef]

del Barrio Castro, Tomás, Paulo M. M. Rodrigues, and A. M. Robert Taylor. 2018. Semi-Parametric Seasonal Unit Root Tests. Econometric Theory 34: 447-76. [CrossRef]

del Barrio Castro, Tomás, Paulo M. M. Rodrigues, and A. M. Robert Taylor. 2019. Temporal Aggregation of Seasonally Near-Integrated Processes. Journal of Time Series Analysis 40: 872-86. [CrossRef]

Engle, Robert F., and Clive W. J. Granger. 1987. Co-integration and error correction: Representation, estimation and testing. Econometrica 55: 251-76. [CrossRef]

Entorf, Horst. 1997. Random walks with drifts: Nonsense regression and spurious fixed-effect estimation. Journal of Econometrics 80: 287-96. [CrossRef]

Fu, Ke-Ang, and Xiaoyong Fu. 2015. Asymptotics for the random coefficient first-order autoregressive model with possibly heavy-tailed innovations. Journal of Computational and Applied Mathematics 285: 116-24. [CrossRef]

Ghysels, Eric, and Denise R. Osborn. 2001. The Econometric Analysis of Seasonal Time Series. Cambridge : Cambridge University Press.

Granger, Clive. 1981. Some properties of time series data and their use in econometric model specification. Journal of Econometrics 16: 121-30. [CrossRef]

Granger, Clive W. J., and Paul Newbold. 1974. Spurious Regressions in Econometrics. Journal of Econometrics 2: 111-20. [CrossRef]

Johansen, Søren, and Ernst Schaumburg. 1999. Likelihood analysis of seasonal cointegration. Journal of Econometrics 88: 301-39. [CrossRef]

Pesaran, M. Hashem, Yongcheol Shin, and Ron J. Smith. 1999. Pooled mean group estimation of dynamic heterogeneous panels. Journal of the American Statistical Association 94: 621-34. [CrossRef]

Phillips, Peter C. B. 1986. Understanding spurious regressions in econometrics. Journal of Econometrics 33: 311-40. [CrossRef]

Westerlund, Joakim. 2008. Panel cointegration tests of the Fisher effect. Journal of Applied Econometrics 23: 193-233. [CrossRef]

Pötzelberger, Klaus. 1990. A characterization of random-coefficient AR(1) models. Stochastic Processes and Their Applications 34: 171-80. [CrossRef]

Smith, Richard J., A. M. Robert Taylor, and Tomas del Barrio Castro. 2009. Regression-Based Seasonal Unit Root Tests. Econometric Theory 25: 527-60. [CrossRef]

Tiku, Moti L., and Wing-Keung Wong. 1998. Testing for unit root in AR(1) model using three and four moment approximations. Communications in Statistics: Simulation and Computation 27: 185-98. [CrossRef]

Tiku, Moti L., Wing Keung Wong, and Guorui Bian. 1999. Time series models with asymmetric innovations. Communications in Statistics: Theory and Methods 28: 1331-60. [CrossRef] 
Tiku, Moti L., Wing-Keung Wong, David C. Vaughan, and Guorui Bian. 2000. Time series models in non-normal situations: Symmetric innovations. Journal of Time Series Analysis 21: 571-96. [CrossRef]

Ventosa-Santaulária, Daniel. 2009. Spurious Regression. Journal of Probability and Statistics 1: 1-27. [CrossRef]

Wong, Wing-Keung, and Guorui Bian. 2005. Estimating Parameters in Autoregressive Models with asymmetric innovations. Statistics and Probability Letters 71: 61-70. [CrossRef] 